\title{
TREM2 activation on microglia promotes myelin debris clearance and remyelination in a model of multiple sclerosis
}

\author{
Francesca Cignarella ${ }^{1,10} \cdot$ Fabia Filipello $^{1,2} \cdot$ Bryan Bollman $^{1} \cdot$ Claudia Cantoni $^{1} \cdot$ Alberto Locca $^{1} \cdot$ Robert Mikesell $^{1}$. \\ Melissa Manis ${ }^{1} \cdot$ Adiljan Ibrahim $^{3} \cdot$ Li Deng $^{1,4} \cdot$ Bruno A. Benitez $^{5,6,7} \cdot$ Carlos Cruchaga ${ }^{5,6,7}$. Danilo Licastro ${ }^{8}$. \\ Kathie Mihindukulasuriya ${ }^{5,7}$. Oscar Harari ${ }^{5,6,7}$. Michael Buckland ${ }^{9}$. David M. Holtzman ${ }^{1,6}$. Arnon Rosenthal ${ }^{3}$. \\ Tina Schwabe ${ }^{3} \cdot$ Ilaria Tassi $^{3} \cdot$ Laura Piccio $^{1,7,9}$
}

Received: 14 March 2020 / Revised: 1 July 2020 / Accepted: 6 July 2020 / Published online: 9 August 2020

(c) The Author(s) 2020

\begin{abstract}
Multiple sclerosis (MS) is an inflammatory, demyelinating, and neurodegenerative disease of the central nervous system (CNS) triggered by autoimmune mechanisms. Microglia are critical for the clearance of myelin debris in areas of demyelination, a key step to allow remyelination. TREM2 is expressed by microglia and promotes microglial survival, proliferation, and phagocytic activity. Herein we demonstrate that TREM2 was highly expressed on myelin-laden phagocytes in active demyelinating lesions in the CNS of subjects with MS. In gene expression studies, macrophages from subjects with TREM2 genetic deficiency displayed a defect in phagocytic pathways. Treatment with a new TREM 2 agonistic antibody promoted the clearance of myelin debris in the cuprizone model of CNS demyelination. Effects included enhancement of myelin uptake and degradation, resulting in accelerated myelin debris removal by microglia. Most importantly, antibody-dependent TREM2 activation on microglia increased density of oligodendrocyte precursors in areas of demyelination, as well as the formation of mature oligodendrocytes thus enhancing remyelination and axonal integrity. These results are relevant as they propose TREM2 on microglia as a potential new target to promote remyelination.
\end{abstract}

\section{Introduction}

Francesca Cignarella and Fabia Filipello contributed equally.

Electronic supplementary material The online version of this article (https://doi.org/10.1007/s00401-020-02193-z) contains supplementary material, which is available to authorized users.

Ilaria Tassi

Ilaria.tassi@alector.com

$\triangle$ Laura Piccio

picciol@wustl.edu; laura.piccio@sydney.edu.au

1 Department of Neurology, Washington University School of Medicine, 660 S. Euclid Avenue, Campus Box 8111, St. Louis, MO 63110, USA

2 Department of Biomedical Sciences, Humanitas University, Via Rita Levi Montalcini 4, Pieve Emanuele - Milan 20090, Italy

3 Alector, 131 Oyster Point Blvd \#600, South San Francisco, CA 94080, USA

4 Department of Anesthesiology, First Affiliated Hospital of Soochow University, Suzhou 215006, Jiangsu, China
Multiple sclerosis (MS) is a chronic demyelinating disease of the central nervous system (CNS), whose pathogenesis involves inflammatory and neurodegenerative processes. Current MS immunomodulatory treatments target CNS inflammation, while therapies capable of regenerating

5 Department of Psychiatry, Washington University School of Medicine, St. Louis, MO 63110, USA

6 Hope Center for Neurological Disorders, Washington University School of Medicine, St. Louis, MO 63110, USA

7 NeuroGenomics and Informatics, Washington University School of Medicine, St. Louis, MO 63110, USA

8 ARGO Open Lab Platform for Genome sequencing, AREA Science Park, Padriciano 99, 34149 Trieste, Italy

9 Brain and Mind Centre, University of Sydney, 94 Mallett St Camperdown, Sydney, NSW 2050, Australia

10 Present Address: Alector, 131 Oyster Point Blvd \#600, South San Francisco, CA 94080, USA 
myelin and halting disease progression are lacking [28]. Oligodendrocyte precursor cells (OPCs) are multipotent progenitor cells widely distributed in the CNS that could differentiate into mature oligodendrocytes (OLs) to sustain remyelination. In MS, impaired generation of OLs from OPCs leads to persistent demyelination, myelin debris accumulation, and axonal damage which clinically manifests as neurological disability [11]. Efficient myelin debris removal and clearance by phagocytic cells are critical to eliminate inhibitory signals interfering with OPC activation, recruitment to the site of demyelination and/or differentiation into myelinating mature OLs [14, 26, 27].

Microglial cells and infiltrating monocytes/macrophages can have a dual role in MS lesions. They could contribute to myelin damage and lesion expansion, or they may have a protective role by clearing myelin debris, reducing inflammation and secreting regenerative factors promoting remyelination [48]. A critical modulator of microglia functions is the triggering receptor expressed on myeloid cells-2 (TREM2), an innate immune receptor expressed by several myeloid cells including brain microglia [23, 45]. TREM2 is a phospholipid sensing receptor known to sustain microglial cell activation and expansion in response to demyelination or amyloid plaques in Alzheimer's disease (AD) [8, 42, 50]. Recently, TREM2 was proposed to be a key transcriptional regulator of cholesterol metabolism during chronic phagocytic activity for myelin clearance in response to demyelination [35].

TREM 2 binds on the membrane to the DNAX-activation protein 12 (DAP12) which is an adaptor protein required for TREM2 surface expression and intracellular signaling [47]. TREM2 engagement leads to DAP12 phosphorylation, followed by recruitment and activation of the spleen-associated tyrosine kinase (SYK), resulting in downstream signaling events leading to proliferation, survival, phagocytosis, and secretion of cytokines and chemokines [47]. Homozygous loss-of-function mutations in TREM2 or DAP12 genes cause Nasu-Hakola disease (NHD), a rare genetic disorder characterized by fatal presenile dementia and bone cysts $[38,39]$. Neuropathological findings in NHD include loss of myelin and axons in the brain, with reactive astrocytosis and microglial activation [25]. Heterozygous TREM2 gene variants were reported to increase the risk for $\mathrm{AD}$ and other neurodegenerative diseases (frontotemporal dementia, Parkinson's disease and amyotrophic lateral sclerosis) [7, 16, 20, 43].

Here, we explored the effect of antibody-mediated TREM2 activation on microglia in a well-established toxininduced model of demyelination in the CNS resulting from exposure to the copper chelator cuprizone (CPZ) in the diet [31]. In this model, oligodendrocyte degeneration in the brain is followed by a robust microglial response consisting of activation, proliferation and clearance of myelin debris [17]. These events lead to OPC recruitment, differentiation into mature OLs, and then remyelination which is almost complete within few weeks from toxin withdrawal [31]. We show that treatment with a TREM2-agonistic antibody was able to enhance myelin debris clearance by microglia in vivo in the CPZ-model and by bone marrow-derived macrophages (BMDM) in vitro. Most importantly, these events resulted in increased OPC recruitment and differentiation into mature OLs eventually accelerating remyelination in vivo and preserving axonal health. This provides the proof of concept that antibody-mediated TREM 2 activation could promote remyelination, suggesting this as a potential novel therapeutic avenue in MS and other demyelinating disorders.

\section{Materials and methods}

\section{Mice}

Trem $2^{+/+}$, Trem $2^{+/,}$, and Trem $2^{-/-}$mice (backcrossed 12 generations to the C57BL/6 background) were obtained from Professor Marco Colonna (Washington University in St. Louis). These three strains were bred in parallel. Animals were housed in accordance with University and National Institutes of Health (NIH) guidelines, and animal protocols were approved by the Washington University Animal Studies Committee (study approval number: 20180056). Mice were maintained under controlled conditions $\left(19-22{ }^{\circ} \mathrm{C}\right.$ and a in a 12-h light/dark cycle with unrestricted access to food and water). Experiments performed by Alector (Fig. 2 and Supplementary Fig. 1a and c) used the Trem $2^{-/-}$mouse colony originally generated by the trans-NIH KnockOut Mouse Project (KOMP). Frozen sperms were obtained from the UC Davis KOMP repository, and a colony of mice was established at UC Davis.

\section{Antibody generation}

Monoclonal antibodies targeting mouse TREM 2 were generated by immunizing mice genetically deficient of TREM 2 $\left(\right.$ Trem $2^{-1}$ ) with recombinant TREM2 protein and hybridoma generation, as well as by a yeast display campaign (performed by Adimab). AL002a was screened for TREM2 specificity by selecting for binding to wild-type $\left(\right.$ Trem $\left.^{+/+}\right)$, but not Trem2 $2^{-/-}$BMDM.

\section{Mouse model of CPZ-induced demyelination}

Six- to eight-week-old $\operatorname{Trem} 2^{+/+}, \operatorname{Trem} 2^{+/-}$, and Trem $2^{-/-}$mice were fed a $0.2 \%$ Bis-(cyclohexanone) oxaldihydrazone (cuprizone) diet (5C5N: Modified PicoLab ${ }^{\circledR}$ Rodent w/0.2\% Cuprizone, TestDiet. Cuprizone from Alpha Aesar, A10628) for 4 weeks (WK 4) or for 4 weeks followed by 3 days (WK $4+3 \mathrm{D}$ ), 7 days (WK $4+7 \mathrm{D}$ ), or 14 days 
(WK 4 + 14D) on regular chow (PicoLab Rodent Diet 20 \#5053, Purina). For the full duration of the experiment mice were injected intraperitoneally (i.p.) once a week with the anti-TREM2 antibody or the control antibody at a dose of $80 \mathrm{mg} / \mathrm{kg}$. The first injection with the antibodies was performed 4 days before beginning the CPZ-diet.

\section{Quantification of antibody levels in the brain}

Six- to eight-week-old Trem $2^{+/-}$mice were injected i.p. with AL002a. $48 \mathrm{~h}$ post injection, brains were removed after perfusion with PBS, micro-dissected to isolate the corpus callosum (CC) and the cortex (Ctx), and immediately frozen in liquid nitrogen. Tissues were solubilized in N-Per lysis buffer (87792, ThermoFisher) and cell protein content was measured using the Pierce bicinchoninic acid (BCA) protein assay kit (23227, Thermo Scientific). A mouse Trem-2b/Fc Chimera (R\&D Systems) was used as a capture antibody and coated overnight at $4{ }^{\circ} \mathrm{C}$ on 96 -well Meso Scale Discovery (MSD) plates in PBS. After washing, wells were blocked for $1 \mathrm{~h}$ at $37{ }^{\circ} \mathrm{C}$ with binding buffer (3\% BSA in PBS). Titration of samples and standards were incubated for $1 \mathrm{~h}$ at room temperature (RT) on a shaker at $500 \mathrm{rpm}$. For detection $(0.5 \mathrm{mg} / \mathrm{ml})$ of Sulfo-TAG goat anti-mouse IgG (MSD) were added to the plate. After washing, read buffer was added and the plate was read on the Sector Imager. Washes between the different steps were done three times with $0.05 \%$ Tween 20 in PBS. Antibody content was normalized to serum and protein content.

\section{Bone marrow-derived macrophages}

Bone marrow-derived macrophages (BMDM) were obtained by flushing tibial and femoral marrow cells with cold PBS 2\% FBS. Red blood cells were lysed using ACK lysing buffer (Thermo Fisher), and after two washes in PBS 2\% FBS, the cells were re-suspended in complete media (RPMI, $10 \%$ FBS, Pen/Strep, L-glutamine, non-essential amino acid) with $(50 \mathrm{ng} / \mathrm{ml})$ murine M-CSF (m-M-CSF) to obtain differentiated macrophages after 6 days. Adherent macrophages were detached with $1 \mathrm{mM}$ EDTA in PBS.

\section{Immunoprecipitation}

Immunoprecipitation in vitro: before stimulation, BMDM were starved for $4 \mathrm{~h}$ in RPMI with $1 \% \mathrm{FBS}$. $10 \times 10^{6}$ cells were incubated for $15 \mathrm{~min}$ at $4{ }^{\circ} \mathrm{C}$ with AL002a or control antibody ( $1 \mu \mathrm{g}$ for $10^{6}$ cells). Cells were then washed and incubated at $37{ }^{\circ} \mathrm{C}$ in the presence of goat-anti mouse $\operatorname{IgG}\left(1.5 \mu \mathrm{g}\right.$ for $1 \times 10^{6}$ cells). After stimulation, cells were lysed with lysis buffer ( $1 \%$ n-dodecyl- $\beta$-D-Maltoside, $50 \mathrm{Mm}$ Tris- $\mathrm{HCl}$ (pH 8.0), $150 \mathrm{mM} \mathrm{NaCl}, 1 \mathrm{mM}$ EDTA, $1.5 \mathrm{mM}$ $\mathrm{MgCl} 2,10 \%$ glycerol, protease, and phosphatase inhibitors) and immunoprecipitated with an anti-TREM2 antibody that binds a different domain (rat anti-h/m-TREM2, clone 237920, R\&D system). Immunoprecipitation in vivo: 6- to 8-week-old C57BL/6 mice were injected i.p. with $3 \mathrm{ml}$ of $3 \%$ thioglycollate. After 3 days, when the peritoneal cavity was enriched with $\mathrm{CD} 11 \mathrm{~b}^{+} \mathrm{F} 4 / 80^{+}$macrophages expressing TREM2, mice were injected with control or TREM2-specific antibodies $(40 \mathrm{mg} / \mathrm{kg}) .24 \mathrm{~h}$ after antibody injection, peritoneal macrophages were collected, immediately lysed in the lysis buffer previously described, and immunoprecipitated with rat anti-h/m TREM2 antibody described above (R\&D System, clone 237920). Then, for both in vitro and in vivo experiments, precipitated proteins were fractionated by SDS-PAGE in non-reducing conditions, transferred to PVDF membranes, and probed with an anti-phosphotyrosine antibody (4G10, Millipore). TREM2 is not detected in nonreducing condition. To confirm that all substrates were adequately immunoprecipitated, whole cell lysates from each sample were also fractionated by SDS-PAGE in reducing condition and immunoblotted with an anti-actin antibody (actin, sc-47778 Santa Cruz).

\section{Myelin production}

Human myelin was prepared as previously described [34] and stored in lyophilized form at $-80{ }^{\circ} \mathrm{C}$. Prior to use, myelin was suspended in DMEM to a final concentration of ( $2 \mathrm{mg} / \mathrm{ml}$ ) and dissolved by vortexing and sonicating. Myelin was then irradiated with 10,000 RADS to achieve sterility. Aliquots were stored at $-80^{\circ} \mathrm{C}$ for further use.

\section{NFAT-Luciferase reporter assay}

A stable BW5147.G.1.4 (ATCC ${ }^{\circledR}$ TIB48 $^{\mathrm{TM}}$ ) (BWZ) cell line expressing both mouse TREM2 and DAP12 was generously provided by the Seaman lab [10]. This line was infected with a Cignal PLenti NFAT-Luciferase virus (Qiagen) to generate a stable mouse TREM2 reporter cell line, able to induce luciferase signaling upon TREM2 activation. The activity of the reporter was validated using PMA $(0.05 \mu \mathrm{g} /$ $\mathrm{ml})$ and ionomycin $(0.25 \mu \mathrm{M})$. To test whether TREM2 antibody induced signaling, $5 \mu \mathrm{g} / \mathrm{ml}$ of soluble AL002a, or control antibody was added to each well of 96-well culture plates together with 100,000 cell/well and incubated for $4-6 \mathrm{~h}$ at $37{ }^{\circ} \mathrm{C}$ in Dulbecco's Modified Eagle Medium (DMEM). Luciferase activity was measured by removing media and adding $50 \mu \mathrm{l}$ of PBS and $50 \mu \mathrm{l}$ of OneGlo Reagent (Promega) to each well and incubating for $3 \mathrm{~min}$ at room temperature on a plate shaker to lyse the cells. Luciferase signal was measured using a BioTek plate reader. Data were analyzed using GraphPad Prism.

To test myelin-induced signaling, human myelin was diluted in PBS to $200 \mu \mathrm{g} / \mathrm{ml}$, titrated onto a 96-well tissue 
culture plate, and incubated overnight at $4{ }^{\circ} \mathrm{C}$. The next morning, the solution was removed, and plates were washed three times with $200 \mu \mathrm{l}$ PBS. Plates were air dried and BWZ cells (with or without antibodies) were added, incubated, and analyzed as described above.

\section{Myelin phagocytosis and degradation assays}

BMDM obtained from Trem $2^{+/-}$mice were seeded in 8-well chamber slides (35,000 cells/well) (154534 Nunc, Lab-Tek) in normal medium as described before. After 1 day, media was substituted with reduced FBS media (5\% FBS) and macrophages were pre-incubated with AL002a or control antibody $(10 \mu \mathrm{g} / \mathrm{ml}) 3 \mathrm{~h}$ before starting the experiments. For phagocytosis assays, BMDM were incubated with human myelin $(20 \mu \mathrm{g} / \mathrm{ml})$ for $30 \mathrm{~min}, 1 \mathrm{~h}$, or $3 \mathrm{~h}$, and after incubation, cells were washed in PBS and fixed in $4 \%$ PFA. For degradation assays, BMDM were incubated with human myelin $(20 \mu \mathrm{g} / \mathrm{ml})$ for $2 \mathrm{~h}$, then washed thoroughly with PBS, and again put in culture in reduced FBS media. Cells were left in culture for $1 \mathrm{~h}, 24 \mathrm{~h}$, or $48 \mathrm{~h}$, then washed in PBS, and fixed in 4\% PFA. For intracellular staining, cells were permeabilized and blocked for $60 \mathrm{~min}$ at RT in $5 \%$ horse serum and $0.1 \%$ saponin in PBS, and incubated at $4{ }^{\circ} \mathrm{C}$ overnight with primary antibodies Rt anti-MBP (Abcam, ab7349, 1:100) and Gt anti-Iba1 (Novus, NB100-1028, 1:250) diluted in PBS and 5\% horse serum.

\section{Mouse tissue processing and histological analyses}

Mice were perfused with $4 \%$ paraformaldehyde. Mouse brains were removed and post-fixed in $4 \%$ PFA for $24 \mathrm{~h}$, followed by immersion in $30 \%$ sucrose for $48 \mathrm{~h}$, then embedded in Optimal Cutting Temperature (OCT). 5- $\mu \mathrm{m}$ sections were placed on glass slides and stained with solochrome cyanine to confirm the presence of a lesion as previously described [22]. Sections were stained with the following primary antibodies: Rb anti-dMBP (Millipore, ab5864, 1:2000), Rb anti-Iba1 (Wako, 019-19741, 1:600), Gt anti-Iba1 (Novus, NB100-1028, 1:250), Rt anti-LAMP1 (Abcam, ab25245, 1:500), Rt anti-CD68 (Invitrogen, 14-0681-82, 1:300), $\mathrm{Rb}$ anti-PDGFR $\alpha$ (ThermoFisher, PA5-16742, 1:50), Rb anti-OLIG2 (Milipore, AB9610, 1:300), Ms anti-CNPase (Abcam, ab6319, :100), Shp anti-BrdU (Abcam, ab1893, 1:250), Rt anti-GFAP (ThermoFisher, 13-0300, 1:200), and Ms anti-SMI-31 (Biolegend, 801603, 1:1000). AlexaFluorconjugated secondary antibodies (Invitrogen, 1:1000) were used. Some of the images were acquired with a Nikon Eclipse $90 i$ fluorescent and bright field microscope equipped with $10 \times$ and $20 \times$ zoom objectives and analyzed with Metamorph 7.7 software. CNPase, dMBP, and GFAP were analyzed as the percentage area of positive staining (number of positive pixels $/ \mathrm{mm}^{2}$ ) within the region of interest.
Iba1, PDGFR $\alpha, \operatorname{BrdU}$, and OLIG2 were quantified as the density of cells in the region of interest (number of cells/ $\mathrm{mm}^{2}$ ). LAMP1 and CD68 were analyzed as the percentage area of $\mathrm{LAMP}^{+} \mathrm{Iba}^{+}$and $\mathrm{CD}^{+} 8^{+} \mathrm{Iba}^{+}{ }^{+}$staining (number of positive pixels $/ \mathrm{mm}^{2}$ ) and then normalized on the percentage of $\mathrm{Iba}^{+}$staining (number of positive pixels $/ \mathrm{mm}^{2}$ ) within the region of interest. For confocal analysis, images were acquired with an Olympus FV1200 laser scanning confocal microscope (Olympus-America Inc., Waltham, MA) equipped with a PlanApoN $60 \times, 1.4$ NA super corrected oil objective. The Olympus FV1200 confocal microscope was equipped with five detectors: two spectral and one filterbased and two gallium arsenide phosphide (GaAsP) photomultiplier tubes (PMTs). The 405-, 488-, and 559-nm diode lasers and 635-nm HeNe (helium neon) lasers were used with an optimal pinhole of 1 airy unit to acquire images. Images were finally processed with ImageJ and Imaris Software (Bitplane, Switzerland).

\section{Transmission electron microscopy and g-ratio quantification}

Mice were perfused with PBS, brains were removed, and immersion fixed in 2\% PFA, $2.5 \%$ Glutaraldehyde, and $0.1 \mathrm{M}$ PBS. $50 \mu \mathrm{m}$ sagittal brain sections were cut using a vibratome, then fixed in osmium tetroxide in 0.1 M PBS (EMS, 19100), followed by dehydration in ethanol and infiltration of Spurr's resin. Tissues were embedded using Spurr's resin and aclar film. After polymerizing, the corpus callosum was dissected from the tissue and attached to a premade Spurr's resin block, then sectioned using a DiATOME ultra $45^{\circ}$ diamond knife and a LEICA Ultracut UC7. 90-nm sections were cut and picked up onto 200 hex mesh, formvarcarbon coated copper grids (Ted Pella, 01800-F), and stained with uranyl acetate and lead citrate. Images were captured using a JEOL 1200 EX II Transmission Electron Microscope with AMT digital camera. Remyelination was analyzed by counting the number of naked axons and the number of myelinated axons per field, with a minimum of ten fields being analyzed. The g-ratio was quantified by dividing the axonal diameter by the myelinated fiber diameter. Thirty myelinated axons were randomly analyzed across multiple fields per mouse to calculate the g-ratio.

\section{In vivo myelin engulfment quantification}

Fixed brain slices were permeabilized for $45 \mathrm{~min}$ at RT in PBS $0.1 \%$ Triton X-100, followed by $1 \mathrm{~h}$ RT in blocking solution (2\% BSA $0.1 \%$ Triton X-100 in PBS) and overnight incubation with primary antibody for $\mathrm{Rb}$ anti-dMBP (Millipore, ab5864, 1:2000), Gt anti-Iba1 (Novus, NB1001028, 1:250) and Rt anti-CD68 (Invitrogen, 14-0681-82, $1: 300)$ at $4{ }^{\circ} \mathrm{C}$. Upon washing, sections were incubated $2 \mathrm{~h}$ 
at RT with Alexafluor-conjugated secondary antibodies (Invitrogen, 1:1000). Images were acquired with an Olympus FV1200 laser scanning confocal microscope (OlympusAmerica Inc., Waltham, MA) with $2 \times$ digital zoom, and a Z-step size of $0.33 \mu \mathrm{m}$. Z-stacks ranged from 4 to $5 \mu \mathrm{m}$ in thickness. Images were processed and analyzed by Imaris Software (Bitplane, Switzerland). CD68 and Iba1 volume was quantified by applying 3D surface rendering of confocal z-stacks in their respective channels, using identical settings (fix thresholds of intensity and voxel) within each experiment. Each confocal acquisition contained an equal number of images from the CC of mice treated with AL002a and control antibody. For quantification of dMBP engulfment by microglia, only dMBP signals present within microglial CD68 ${ }^{+}$structures were considered. To this end, a new channel for "engulfed dMBP" was created, by using the mask function in Imaris, masking the dMBP signal within $\mathrm{CD} 68^{+}$ structures. Quantification of volumes for 'engulfed dMBP in CD68' was performed following the '3D Surface rendering of engulfed material' protocol previously published [44]. To account for variations in cell size, the amount of 'engulfed dMBP in CD68' was normalized to the total volume of the phagocyte in each field (given by $\mathrm{Iba}^{+}$total volume). Total dMBP volume per field from the same confocal z-stacks was also quantified following the same protocol.

\section{Neurofilament light detection}

Mouse blood was collected into EDTA tubes (Sarstedt 201341102) with a capillary tube (Sarstedt 201278100), spun at $15,000 \times g$ for $7 \mathrm{~min}$ at $4{ }^{\circ} \mathrm{C}$, and the top plasma layer was transferred to a $1.5 \mathrm{ml}$ tube and stored at $-80{ }^{\circ} \mathrm{C}$. Frozen plasma samples were thawed at room temperature, diluted tenfold, and run on a SIMOA HD-X (Quanterix) using the Simoa NF-light advantage kit (Quanterix 103186) according to the manufacturer's protocol.

\section{BrdU used as a marker of proliferation}

Microglia and OPC proliferation in vivo was measured by 5-bromo-2'-deoxyuridine (BrdU) incorporation (Sigma, B5002). BrdU was administered $(25 \mathrm{mg} / \mathrm{kg}$ ) by intra peritoneal injection every $12 \mathrm{~h}$ starting 4 days before collecting the brains.

\section{Microglia isolation and flow cytometry}

Microglia isolation was carried out following a published protocol [3] with modifications. The whole procedure was done on ice with cold buffers and centrifuges at $4{ }^{\circ} \mathrm{C}$. Briefly, anesthetized mice were perfused intracardially with ice-cold Hank's balanced salt solution (HBSS), and the CC and hippocampi were dissected under a stereotactic microscope. The different areas were mechanically homogenized and digested in ice-cold Accutase (Millipore) on a wheel for $20 \mathrm{~min}$ at $4{ }^{\circ} \mathrm{C}$. After spinning the tubes for $1 \mathrm{~min}$ at $2000 \mathrm{~g}$, pellets were resuspended in cold Hibernate buffer (Thermo Fisher). The cell suspension was then transferred to pre-chilled $15 \mathrm{ml}$ tubes and passed through a pre-wet (with Hibernate buffer) $70 \mu \mathrm{m}$ cell strainer (PluriStrainer Mini). Cell suspensions were then spun down at $300 \mathrm{~g}$ for $10 \mathrm{~min}$ and pellets were resuspended in PBS $+2 \%$ FBS, counted, and labeled with a combination of the following conjugated antibodies: CD11b-PeCy7 (clone M1/70), CD45-Alexa 700 (clone 30-F11), P2ry12-PE (clone S16007D), CD80-FITC (clone 16-10A1), CD86-BV421 (clone GL-1), and zombie acqua (Biolegend). Dead cells were excluded by selecting the zombie aqua negative cells. FACS analysis of the microglial profile was performed by gating $\mathrm{CD} 11 \mathrm{~b}^{+} \mathrm{CD} 45^{\text {int }}$ cells. Appropriate $\mathrm{IgG}$ isotype control antibodies were used for all staining. FACS analysis was performed on a FACS Fortessa machine (BD Biosciences), and data were analyzed with FlowJo Software (TreeStar).

\section{Human tissue and analysis}

Twenty fresh-frozen blocks of post-mortem CNS tissue from eight MS patients and four controls with non-neurological diseases were obtained from The Neuroinflammatory Disease Tissue Repository at Washington University St. Louis. Demographic and clinical characteristics of the donors of human brain tissues at the time of collection are indicated in Table 1. $5 \mu \mathrm{m}$ sections were stained with Solochrome Cyanine and Oil Red $\mathrm{O}$ to look at myelin and lipid-laden macrophages, respectively. Active lesions were characterized as tissue areas with marked demyelination and the presence of lipid-laden macrophages. Tissues were then stained with Gt anti-human TREM2 (R\&D Systems, AF1828, 1:200) and Rb anti-Iba1 antibody (Wako, 019-19741, 1:600). AlexaFluorconjugated secondary antibodies were used (Invitrogen, 1:1000). Histological images were acquired using the Nanozoomer microscope at the Hope Center for Neurological Disorders at Washington University. For confocal analysis, images were acquired with a Zeiss LSM880 Airyscan laser scanning confocal microscope (Carl Zeiss Inc., Thornwood, NY) equipped with 63X, 1.4 numerical aperture (NA) Zeiss Plan Apochromat oil objective. The system is equipped with a unique scan head, incorporating a high-resolution galvo scanner along with two PMTs and a 32-element spectral detector. ZEN 2.3 black edition software was used to obtain Z-stacks through the entire height of the cells with confocal Z-slices of $5 \mu \mathrm{m}(63 \times)$ and an interval of $0.347 \mu \mathrm{m}$. Images taken were optimized for 1 airy unit using the 405$\mathrm{nm}$ diode, $488 \mathrm{~nm}$ Argon, and $561 \mathrm{~nm}$ diode and $633 \mathrm{~m}$ $\mathrm{HeNe}$ (helium neon) lasers. In addition, the Airyscan unit provides sub-diffraction limited imaging down to $120 \mathrm{~nm}$ 
Table 1 Human CNS tissues from autopsied multiple sclerosis and healthy control subjects

\begin{tabular}{|c|c|c|c|c|c|c|c|}
\hline Patient ID & Age/gender & $\begin{array}{l}\text { Disease dura- } \\
\text { tion at death }\end{array}$ & $\begin{array}{l}\text { Post mortem } \\
\text { interval }\end{array}$ & Cause of death & MS diagnosis & Tissue & Lesion type \\
\hline 38 & $39 / \mathrm{F}$ & NA & 4 & CNS malignant lymphoma & NA & Cerebellum & Control \\
\hline 10 & $41 / \mathrm{M}$ & NA & 24 & Heart failure & NA & $\begin{array}{l}\text { Spinal cord } \\
\text { Spinal cord } \\
\text { Cerebrum }\end{array}$ & $\begin{array}{l}\text { Control } \\
\text { Control } \\
\text { Control }\end{array}$ \\
\hline 52 & $69 / \mathrm{F}$ & NA & 43 & Sepsis & NA & Cerebrum & Control \\
\hline 32 & $56 / \mathrm{F}$ & NA & 18 & Acute myocardial infarction & NA & $\begin{array}{l}\text { Spinal cord } \\
\text { Cerebrum }\end{array}$ & $\begin{array}{l}\text { Control } \\
\text { Control }\end{array}$ \\
\hline 46 & $77 / \mathrm{F}$ & Unknown & 16 & Not reported & SPMS & Spinal cord & MS NAWM \\
\hline 45 & $66 / \mathrm{F}$ & 32 & 29 & Not reported & SPMS & $\begin{array}{l}\text { Brainstem } \\
\text { Cerebrum }\end{array}$ & $\begin{array}{l}\text { MS NAWM } \\
\text { MS NAWM }\end{array}$ \\
\hline 77 & $41 / \mathrm{F}$ & 15 & 12 & $\begin{array}{l}\text { Complication from Type } 1 \\
\text { diabetes mellitus }\end{array}$ & RRMS & $\begin{array}{l}\text { Brainstem } \\
\text { Spinal cord } \\
\text { Cerebrum }\end{array}$ & $\begin{array}{l}\text { MS NAWM } \\
\text { MS NAWM } \\
\text { MS NAWM }\end{array}$ \\
\hline 40 & $60 / \mathrm{M}$ & 14 & 9 & Respiratory failure & SPMS & Spinal cord & MS active \\
\hline 30 & $54 / \mathrm{F}$ & 17 & 7 & Pneumonia & SPMS & Spinal cord & MS active \\
\hline 71 & $50 / \mathrm{F}$ & 13 & 20 & Not reported & SPMS & $\begin{array}{l}\text { Spinal cord } \\
\text { Spinal cord }\end{array}$ & $\begin{array}{l}\text { MS active } \\
\text { MS active }\end{array}$ \\
\hline 21 & $69 / \mathrm{F}$ & 29 & 6 & Metastatic colon cancer & PPMS & $\begin{array}{l}\text { Spinal cord } \\
\text { Spinal cord }\end{array}$ & $\begin{array}{l}\text { MS active } \\
\text { MS active }\end{array}$ \\
\hline 59 & $54 / \mathrm{F}$ & 22 & 8 & Pneumonia & SPMS & Spinal cord & MS active \\
\hline
\end{tabular}

$N A$ not applicable, SPMS secondary progressive multiple sclerosis, $R R M S$ relapsing remitting multiple sclerosis, $P P M S$ primary progressive multiple sclerosis, $N A W M$ normal appearing white matter

resolution. Quantitative PCR analysis was performed on adjacent tissue sections.

\section{Quantitative PCR}

RNA was extracted using the RNeasy Micro Kit (Qiagen), converted into cDNA using the High-Capacity cDNA Reverse Transcription Kit (Applied Biosystems), and used at 20-40 ng in quantitative real-time PCR (qPCR) analysis. The DDCt method was applied to determine differences in gene expression levels after normalization to the arithmetic mean of GAPDH as internal standards. The TaqMan probes used are the following: for human studies: GAPDH (Hh_99999905_m1) and TREM2 (Hs00219132_ m1); for mouse studies: Gapdh (Mm99999915_g1);
Olig2 (Mm01210556_m1); Mbp (Mm01266402_m1); Cnp (Mm01306641_m1); Mog (Mm00447824_m1); and Plp (Mm01297210_m1). All data are the mean of duplicates, and the standard errors of the mean was calculated between duplicates or triplicates. Real-time PCR was performed using an ABI 7000 Real-Time PCR System (Applied Biosystems).

\section{Human macrophage cultures from Nasu-Hakola disease (NHD) patients}

Peripheral blood mononuclear cells (PBMCs) were purified from peripheral blood samples on Ficoll-Paque PLUS density gradient (Amersham Biosciences, Piscataway, NJ). Samples were from three NHD patients and three control
Table 2 Genetic and demographic characteristics of Nasu-Hakola and healthy control subjects included in gene expression analyses of monocyte-derived macrophages

\begin{tabular}{lllll}
\hline Subject ID & TREM2 genotype & Gender & Age & References \\
\hline NHD1 & C97T; homozygous & F & 46 & {$[4]$} \\
NHD2 & C97T; homozygous & M & 47 & {$[4]$} \\
NHD3 & $482+2$ T $\rightarrow$ C; homozygous & F & 53 & {$[39]$} \\
CTR1 & Normal TREM2 alleles & F & 60 & {$[4]$} \\
CTR2 & Normal TREM2 alleles & M & 38 & NA \\
CTR3 & Normal TREM2 alleles & M & 38 & NA \\
\hline
\end{tabular}

Age was at time of analysis

NHD Nasu-Hakola disease, CTR control/healthy subjects, $N A$ not applicable 
subjects, their characteristics at the time of collection are indicated in Table 2. To generate macrophages, PBMCs were seeded in RPMI without FBS for $2 \mathrm{~h}$, then PBMCs were thoroughly washed with PBS, and cultured in complete media (RPMI, 10\% FBS, Pen/Strep, L-glutamine, non-essential amino acid) supplemented with $(50 \mathrm{ng} / \mathrm{ml})$ of recombinant human M-CSF (300-25, Peprotech) for 7 days at $37{ }^{\circ} \mathrm{C}$ in $5 \% \mathrm{CO}_{2}$. Cells were then harvested and immediately lysed in RLT Buffer; RNA was extracted using the RNeasy Micro Kit (Qiagen).

\section{Microarray processing and analysis}

In order to analyze the Affymetrix HuGene-1_0-st-v1 microarrays, CEL files were uploaded into Thermo Fisher's Transcriptome Analysis Console (TAC) Software. We normalized the data in TAC and did differential expression analysis to compare gene expression in 3 control samples with 3 samples carrying TREM2 homozygous mutations. The differentially expressed genes were graphed in a volcano plot, with the $-\log 10$ of the $P$ value on the $Y$-axis and the fold change on the $X$-axis. A twofold or greater change in gene expression and a $P$ value of 0.05 were considered significant and colored red for up-regulated genes and green for down-regulated genes.

\section{Statistical analysis}

Data are displayed as individual dots and mean \pm SEM. For each graph, the number of observations indicated with " $n$ ", and the number of biological replicates (mice) indicated with " $N$ " can be found in the figure legends. Differences between multiple groups were analyzed by one-way ANOVA and a post hoc test (Tukey's or Sidak's or Dunnett's). Comparisons between two groups following a normal distribution were analyzed using an unpaired $t$ test (two-tail distribution) or a Mann-Whitney test when the distribution was not parametric, as indicated in each figure. Statistical analysis was performed using GraphPad Prism (Graph-Pad Software). Values were considered significant if $P<0.05$.

\section{Results}

\section{TREM2 is highly expressed in active MS lesions}

The general consensus is that TREM2 is expressed by microglia in the CNS, especially after activation [6, 21, 47]. TREM2 expression was reported in active demyelinating lesions on myelin-laden macrophages (called foamy macrophages), which are clearing out myelin debris, a critical a

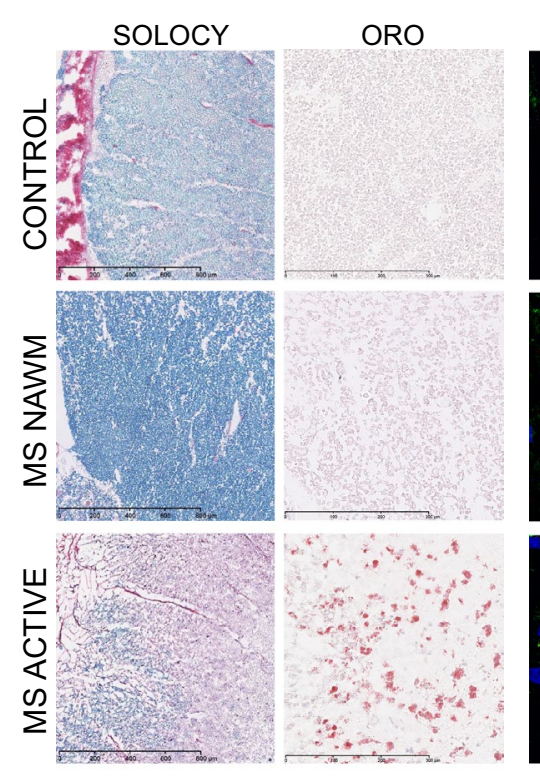

b

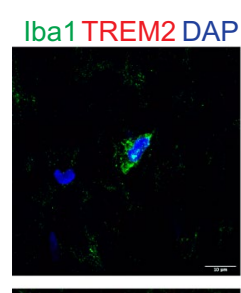

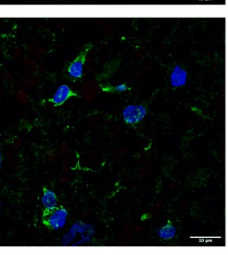

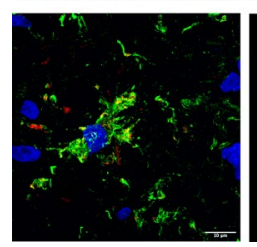

TREM2

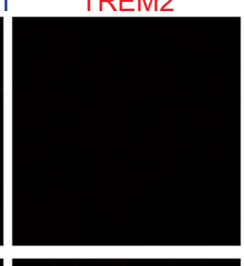

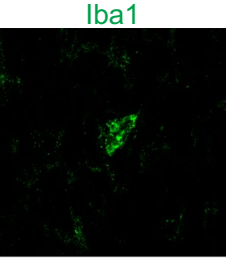
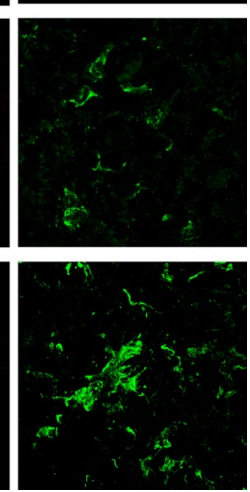

C

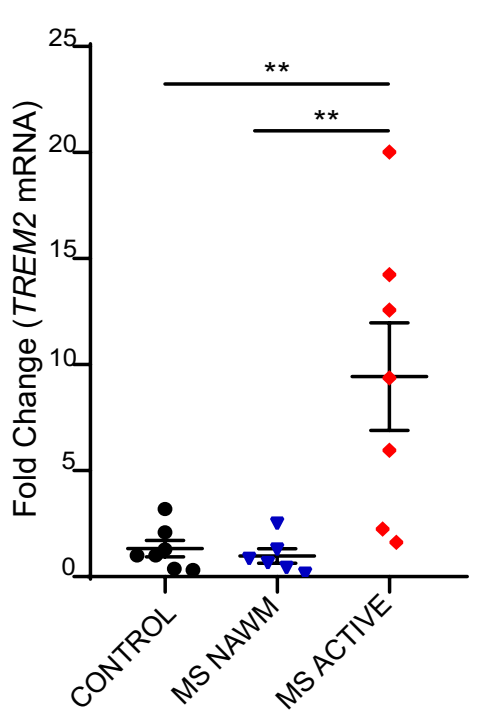

Fig. 1 TREM2 is highly expressed in active MS lesions. a Top to bottom: white matter with no pathology in control non-MS individuals (CONTROL), normal appearing white matter of MS subjects (MS NAWM) and MS active lesions in the white matter (MS ACTIVE) are stained with solochrome cyanine staining (SOLOCY) and with Oil Red O staining (ORO). Original magnification SOLOCY: $\times 10$; scale bar: $800 \mu \mathrm{m}$; ORO: $\times 20$; scale bar: $300 \mu \mathrm{m}$. b Representative confocal images of Iba1 (green), TREM2 (red), and DAPI (blue) in CONTROL, MS NAWM, and MS ACTIVE lesions. Original magnification, $\times 63$. Scale bar, $10 \mu \mathrm{m}$. c Relative TREM 2 mRNA expression by qPCR of the previously described tissue types. 7 samples from 4 CONTROLS, 6 NAWM samples from 3 MS patients, and 7 ACTIVE lesion samples from 5 MS patients. $* * P<0.01$, One-way ANOVA with Tukey's post hoc test 
a

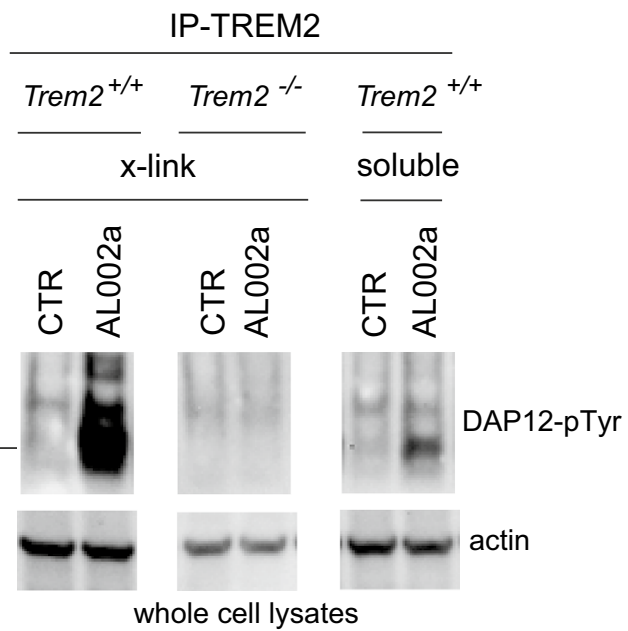

C

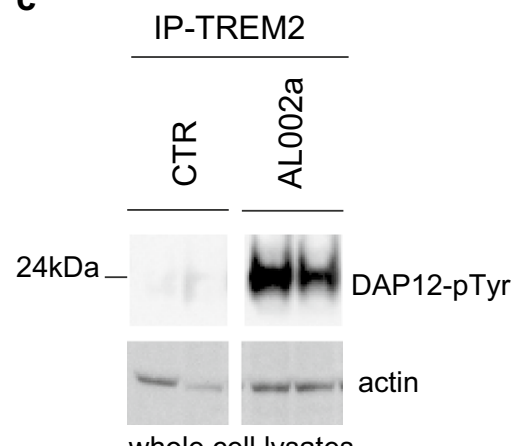

whole cell lysates

f

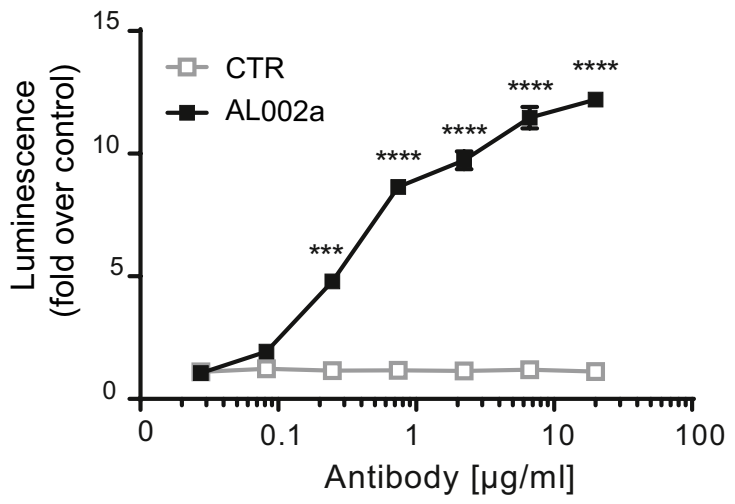

b

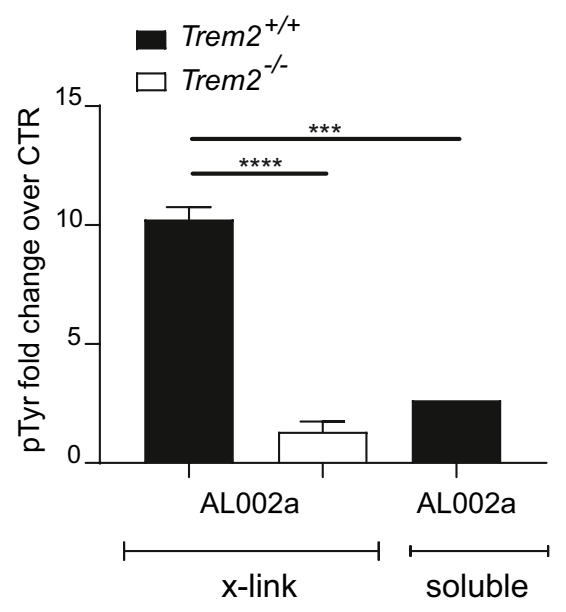

e

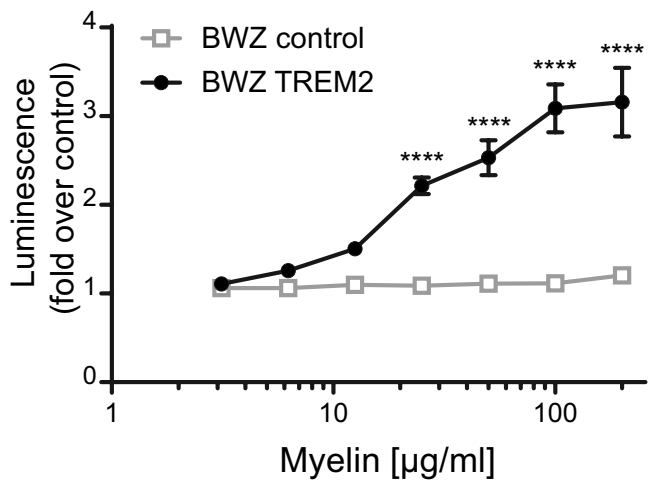

g

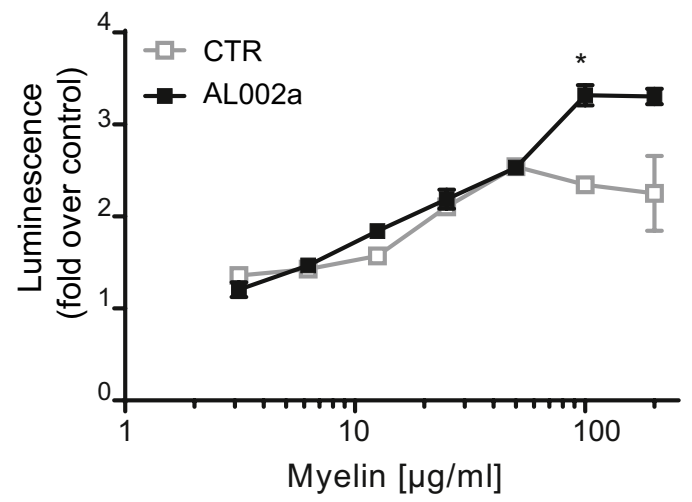

step to allow remyelination [41]. Yet, a systematic characterization of TREM2 expression on microglia-macrophages in different types of MS lesions has not been done. Here we studied TREM2 expression by immunohistochemistry (IHC) and real-time qPCR in human CNS tissues derived from autopsy of subjects with MS or unaffected controls (Table 1). We specifically analyzed MS active lesions and normal appearing white matter (NAWM) in MS subjects and white matter with no pathology in non-MS individuals as control. By confocal analysis we showed TREM2 specific expression in ionized calcium-binding adapter molecule $1(\mathrm{Iba} 1)^{+}$cells in active MS lesion, which were identified as oil-red $\mathrm{O}^{+}$lipid laden macrophages-microglia by histology (Fig. 1a, b). TREM2 was not detectable by IHC on $\mathrm{Iba}^{+}$ microglia in NAWM in MS subjects as well as in the white matter of control individuals (Fig. 1b). 
4Fig. 2 In vitro and in vivo effects of a TREM2-specific antibody on TREM2 signaling. a WB analysis of Trem $2^{+/+}$or Trem $2^{-/-}$BMDM stimulated with a TREM2-specific antibody (AL002a) or with a control antibody, by cross-linking with a secondary antibody (x-link) or in solution (soluble; for Trem $2^{+/+}$only). Cells were immunoprecipitated with a rat anti-h/m-TREM2 antibody and fractionated by SDSPAGE in non-reducing conditions. Phopho DAP12 (pTyr) is shown here as a dimer. b Quantification of DAP12-pTyr normalized on actin is shown as fold change over control. $* * * P<0.001, * * * * P<0.0001$, One-way ANOVA with Tukey's post hoc test. c Peritoneal cells isolated from Trem $^{+/+}$mice after thyoglicollate treatment, immunoprecipitated with an anti-TREM2 antibody (clone 237920), and analyzed by WB. d Quantification of DAP12- pTyr normalized on actin. $* * * * P<0.0001$, two-tailed unpaired Student's $t$ test. e Activation of TREM2 signaling in the BWZ reporter cell assay with different concentrations of plate-bound myelin. BWZ cells express either NFAT:luciferase alone (BWZ control) or in combination with mouse TREM2 and DAP12 (BWZ TREM2). $* * * * P<0.0001$, Two-way ANOVA with Sidak's post hoc test. f TREM2 signaling in the BWZ Trem 2 reporter cell assay at different concentrations of AL002a antibody. $* * * P<0.001, * * * * P<0.0001$, Two-way ANOVA with Dunnett's post hoc test. $\mathbf{g}$ Myelin-induced signaling by AL002a (10 $\mu \mathrm{g} /$ $\mathrm{ml}$ ) in the BWZ Trem2 reporter cell assay at different concentrations of plate-bound myelin. $* P<0.05$, Two-way ANOVA with Dunnett's post hoc test. CTR control

To quantify TREM 2 expression in different lesion types, we performed quantitative real-time RT-PCR (RT-qPCR) studies which demonstrated that TREM2 expression is significantly higher in active MS lesions compared to MS NAWM or white matter from controls (Fig. 1c).

These results provide evidence that TREM2 is highly expressed by Iba $1^{+}$cells present in active demyelinating MS lesions. Therefore, we sought to further evaluate TREM2 potential involvement in the context of CNS demyelination and myelin debris clearance by microglia.

\section{Effects of a newly generated TREM2-specific antibody on TREM2 signaling}

To characterize TREM2 functions on microglia during CNS demyelination, we generated a monoclonal antibody targeting mouse TREM2, called AL002a. The antibody's specificity was verified by binding to wild-type $\left(\right.$ Trem $\left.2^{+/+}\right)$, but not Trem $2^{-/-}$thioglycollate-induced macrophages (THGMac) (Supplementary Fig. 1a). To test whether AL002a induced signaling after receptor engagement, Trem $2^{+/+}$and Trem $^{-/-}$BMDM were stimulated in vitro with AL002a, followed by crosslinking with a secondary antibody to induce receptor clustering. AL002a induced phosphorylation of the TREM2-associated adaptor protein DAP12 in Trem $2^{+/+}$, but not in Trem $2^{-/-}$BMDM (Fig. 2a, b; DAP12 phosphorylation is indicated as DAP12-pTyr). Treatment with an isotype control antibody did not show any DAP12 activation. DAP12 phosphorylation was also induced when BMDM were stimulated with AL002a without secondary crosslinking (soluble) (Fig. 2a, b). These results demonstrate that AL002a is a strong inducer of DAP12 phosphorylation (after cross-linking and as soluble), and it acts as an agonist of TREM2 signaling.

Next, we investigated whether AL002a was able to induce TREM 2 clustering and signaling activation in vivo. Mice were injected with the anti-TREM2 antibody after thioglycollate-induced recruitment of macrophages to the peritoneal cavity. AL002a induced phosphorylation of DAP12-associated TREM2 compared to the isotype control (Fig. 2c, d). These results demonstrate that AL002a is a potent inducer of TREM2 intracellular signaling in vitro (by both cross-linking and soluble stimulation) and in vivo (by soluble stimulation).

It was previously demonstrated that TREM 2 recognizes myelin-associated lipids [42, 52], suggesting that TREM2 may directly sense lipid components exposed after myelin damage. To confirm these results, we developed a cell reporter assay, using BWZ cells expressing the mouse TREM2/DAP12 complex and transduced with a luciferase reporter gene under NFAT promoter control. TREM2 clustering promotes calcium flux stimulating NFAT-activity that induces luciferase expression [50]. In the presence of purified human myelin, TREM2 signaling was activated in the BWZ cell reporter system in a dose-dependent fashion (Fig. 2e). Next, we tested the ability of soluble AL002a to activate TREM2 signaling in the reporter cell assay. AL002a induced TREM2 signaling as demonstrated by incremental luciferase induction in a dose-dependent manner (Fig. 2f). Subsequently, we tested whether TREM2 antibodies could block or enhance myelin-induced TREM 2 signaling. To this end, we added AL002a to BWZ cells seeded on increasing concentrations of plate-bound myelin. At lower myelin concentrations $(<100 \mu \mathrm{g} / \mathrm{ml})$, AL002a antibody did not modulate signaling; in contrast, at higher concentrations (>100 $\mu \mathrm{g} / \mathrm{ml}$ ), AL002a significantly increased myelininduced luciferase activation (Fig. 2g). These results show that AL002a potentiates myelin-mediated TREM2 signaling activation in vitro.

In conclusion, our data demonstrate that AL002a acts as a potent TREM 2 agonist both in vitro and in vivo, and its agonistic activity is boosted by the presence of myelin.

\section{Trem2 haploinsufficiency results in defective myelin debris clearance in the CPZ model of CNS demyelination}

We and others have previously shown that $\operatorname{Trem} 2^{-/-}$mice present a significant defect in clearance of myelin debris in the CPZ-induced model of demyelination compared to Trem $2^{+/+}$mice $[8,42]$. Whether heterozygous $\left(\operatorname{Trem} 2^{+/-}\right)$ mice would present the same or a milder phenotype than Trem $2^{-/-}$mice has not been evaluated before in the CPZ model. Here, Trem $2^{+/+}$, Trem $2^{+/-}$, and Trem $2^{-/-}$mice 

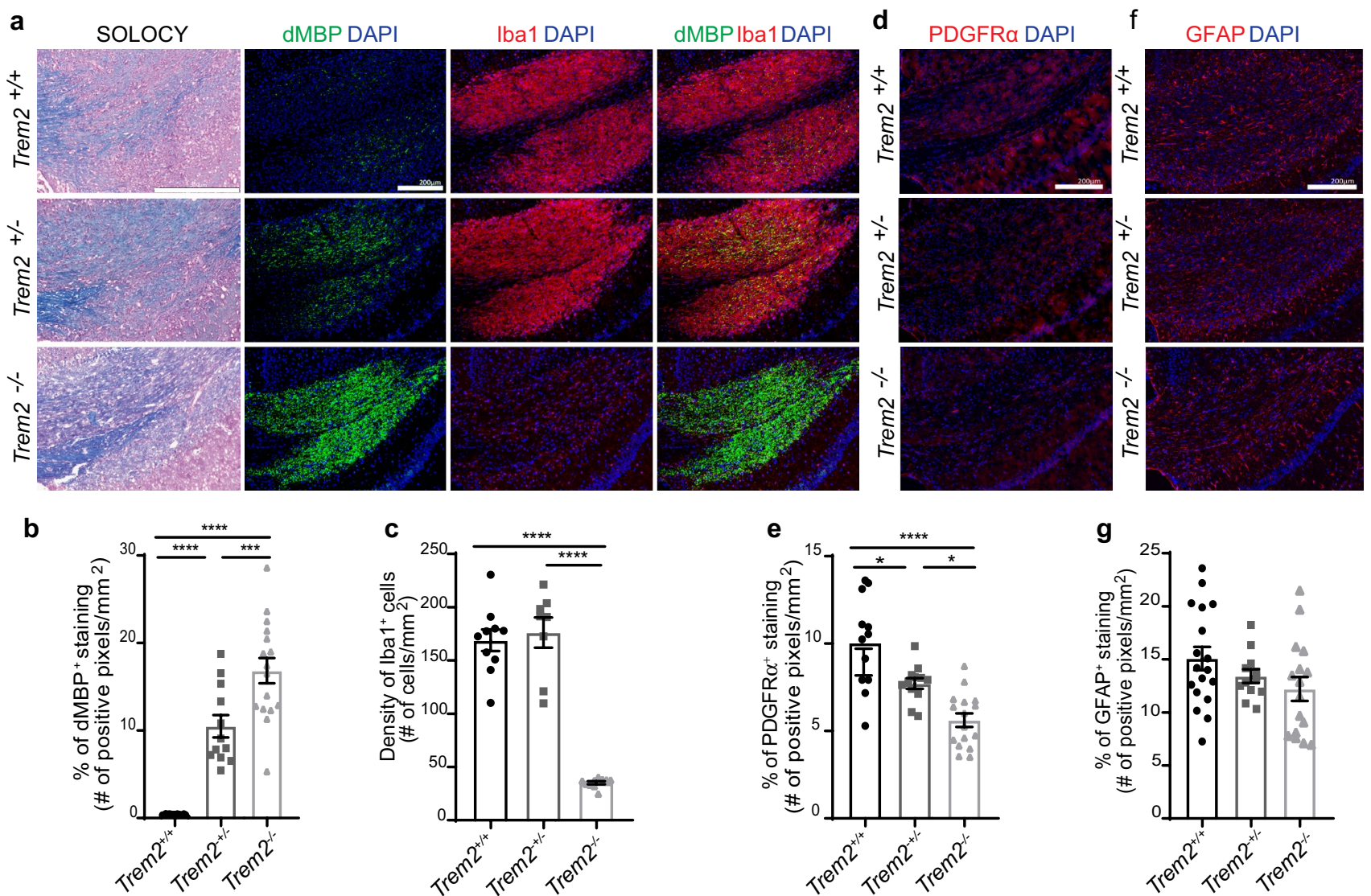

Fig. 3 Trem $2^{+/-}$mice show defective myelin debris clearence after CPZ-induced demyelination. a Representative images of the corpus callosum (CC) of Trem $2^{+/+}$, Trem $2^{+/-}$and Trem $2^{-/-}$mice fed with cuprizone (CPZ) for 4 weeks and then analyzed. Myelin was studied histologically by solochrome cyanine (SOLOCY) staining (intact myelin is stained in dark blue). Scale bar, $300 \mu \mathrm{m}$. dMBP (green) was used to assess myelin debris; microglia were analyzed as density of $\mathrm{Iba}^{+}$cells (red); DAPI (blue). Original magnification, $\times$ 10. Scale bar, $200 \mu \mathrm{m}$. Quantification of $\mathbf{b}$ dMBP fluorescent staining and $\mathbf{c}$ microglia density in the CC after 4 weeks on CPZ. dMBP quantification: $\operatorname{Trem} 2^{+/+} N=8$ mice, $n=16$ fields; Trem $2^{+/-} N=6$, $n=12$; Trem $^{-/-} N=8, n=16$. Iba1 quantification: Trem $2^{+/+} N=5$,

were fed with CPZ for 4 weeks to induce CNS demyelination and to evaluate microglia response to the injury, the degree of myelin debris clearance and remyelination. We performed IHC analyses within the corpus callosum (CC) (Supplementary Fig. 1b), a site of particularly profound and consistent demyelination in the CPZ model [31]. Solochrome cyanine staining was performed to qualitatively visualize myelin in the CC (Fig. 3a). Intact myelin was colored in dark blue in naïve mice (Supplementary Fig. 1c). In contrast, a light blue myelin staining was evident in the CC in Trem $2^{+/-}$, and Trem $2^{-/-}$mice fed CPZ. This was suggestive of tissue accumulation of myelin debris, as we have observed in our previous study [8]. To assess the amount of myelin debris accumulation in $n=10 ;$ Trem $2^{+/-} N=4, n=8 ; \operatorname{Trem} 2^{-/-} N=5, n=10 . * * * P<0.001$, $* * * * P<0.0001$, One-way ANOVA with Tukey's post hoc test. Representative images of PDGFR $\alpha$ (red) (d) and GFAP (red) (f) in the $\mathrm{CC}$ of Trem $2^{+/+}$, Trem $2^{+/-}$and Trem $2^{-/-}$mice after 4 weeks on CPZ. Original magnification, $\times 10$. Scale bar, $200 \mu \mathrm{m}$. Quantification of $\mathbf{e}$ PDGFR $\alpha$ and $\mathbf{g}$ GFAP fluorescent staining in the CC after 4 weeks on CPZ. PDGFR $\alpha$ quantification: Trem $2^{+/+} N=6, n=12$; Trem $2^{+/-}$ $N=6, n=12$; Trem $^{-/-} N=8, n=16$. GFAP quantification: Trem $2^{+/+}$ $N=9, \quad n=18 ;$ Trem $^{+/-} N=6, \quad n=12 ;$ Trem $^{-/-} N=8, n=16$. $* P<0.05, * * * * P<0.0001$, One-way ANOVA with Tukey's post hoc test

the tissue we detected degraded myelin basic protein (dMBP) by using an antibody able to bind MBP epitopes exposed only after myelin degradation [30]. As previously reported, when compared to Trem $2^{+/+}, \mathrm{Trem}^{-/-}$mice after 4 weeks on CPZ displayed a significant increase in dMBP signal, accompanied by a significant reduction in $\mathrm{Iba} 1^{+}$microglia [8] (Fig. 3a-c) and in OPC numbers, identified as Platelet-Derived Growth Factor Receptor Alpha (PDGFR $\alpha)^{+}$cells (Fig. 3d, e). No differences were found in astrocyte activation, quantified as glial fibrillary acidic protein (GFAP) positive cells (Fig. 3f, g). Notably, Trem $2^{+/-}$mice displayed a phenotype which was intermediate between Trem $2^{+/+}$and Trem $2^{-/-}$mice showing a significant increase in dMBP (Fig. 3a, b) and significantly 
a

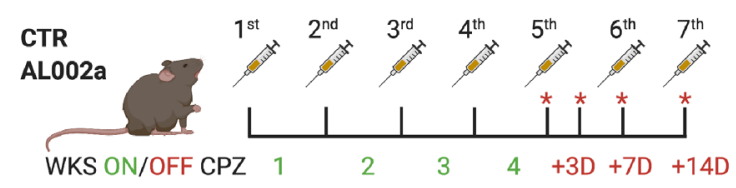

b
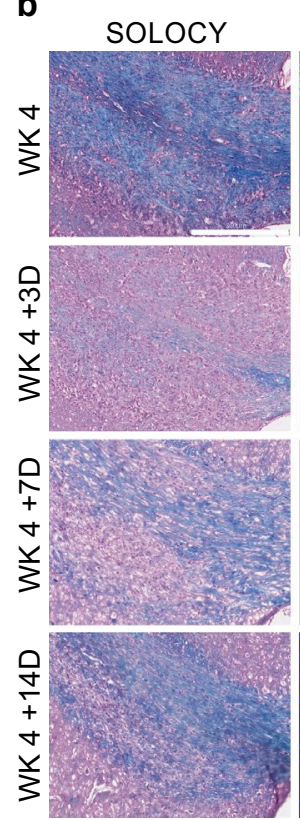

CTR
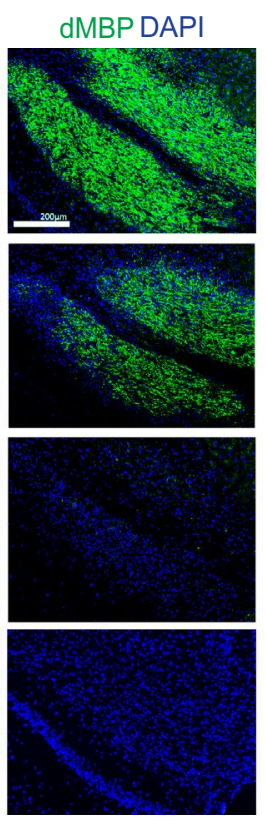

AL002a
SOLOCY
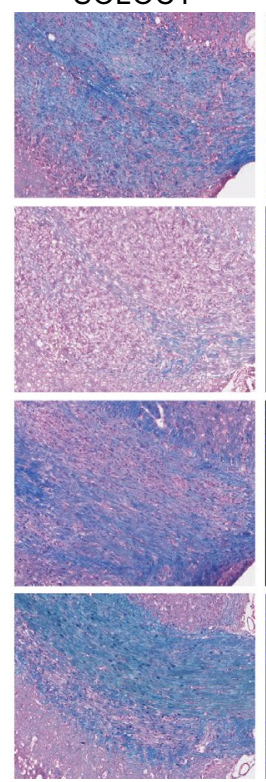

Fig. 4 Treatment with the AL002a antibody during CPZ-induced CNS demyelination in Trem $2^{+/-}$mice promotes myelin debris clearance in the corpus callosum. a Timeline of antibody treatment during cuprizone experiment. Weeks on $\mathrm{CPZ}=\mathrm{WKS} \mathrm{ON}$ (green numbers); days after $\mathrm{CPZ}$ withdrawal $=\mathrm{OFF}$ (red numbers). Mice were sacrificed after 4 weeks on CPZ (WK 4), 4 weeks on CPZ followed by 3 days (+ 3D), 7 days $(+7 \mathrm{D})$ or 14 days $(+14 \mathrm{D})$ of recovery (red asterisks correspond to the time points in which the tissues were collected). b Representative images of solochrome cyanine (SOLOCY) staining and of dMBP (green) and DAPI (blue) immunostaining in the CC of Trem $2^{+/-}$mice at the different time points. Original magnification, $\times 10$. Scale bar, SOLOCY, $300 \mu \mathrm{m}$; dMBP, $200 \mu \mathrm{m}$. c Quan-

less PDGFR $\alpha^{+}$OPCs compared to Trem $2^{+/+}$mice (Fig. 3d, e). No differences were noted in the number of $\mathrm{Iba} 1^{+}$nor $\mathrm{GFAP}^{+}$cells in the CC in Trem $2^{+/-}$compared to Trem $2^{+/+}$ mice (Fig. 3a, c, f, g), in contrast to the significant reduction of the microglia number observed in Trem $2^{-/-}$vs. Trem $2^{+/+}$. These results suggest that the presence of a fully functional TREM2 receptor on microglia is critical for efficient myelin debris removal and quick OPC recruitment observed in Trem $2^{+/+}$mice. Trem 2 haploinsufficiency leads to inefficient myelin debris clearance, reduced OPC recruitment, even if it does not impact on the number of microglia accumulating in the $\mathrm{CC}$.
C
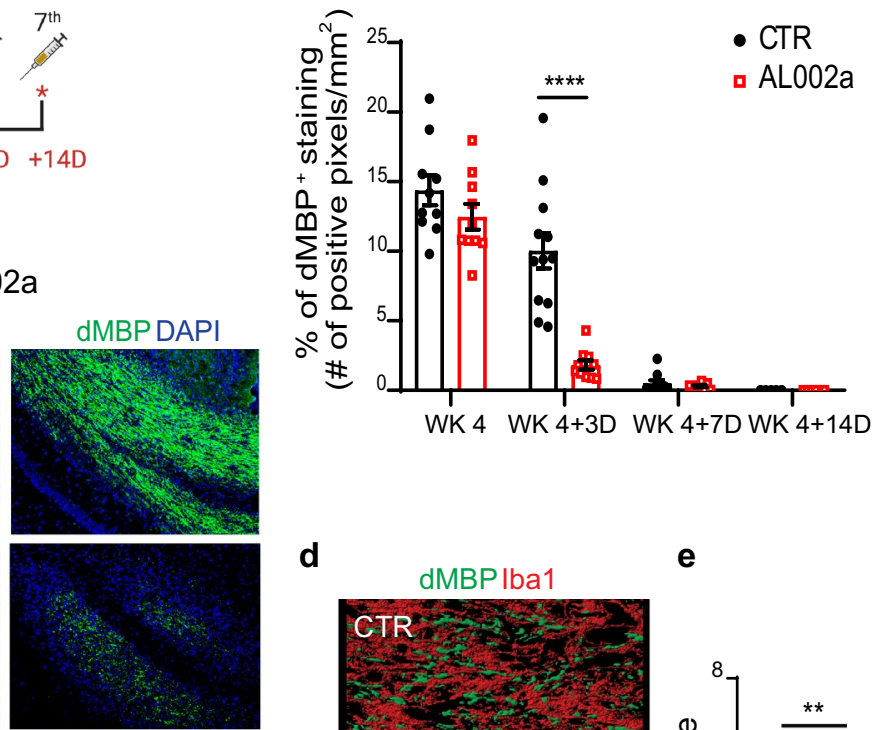

d

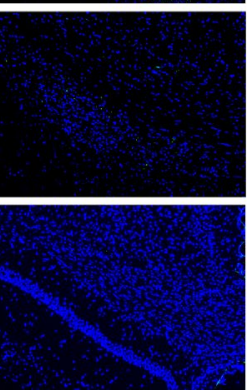

dMBPDAPI
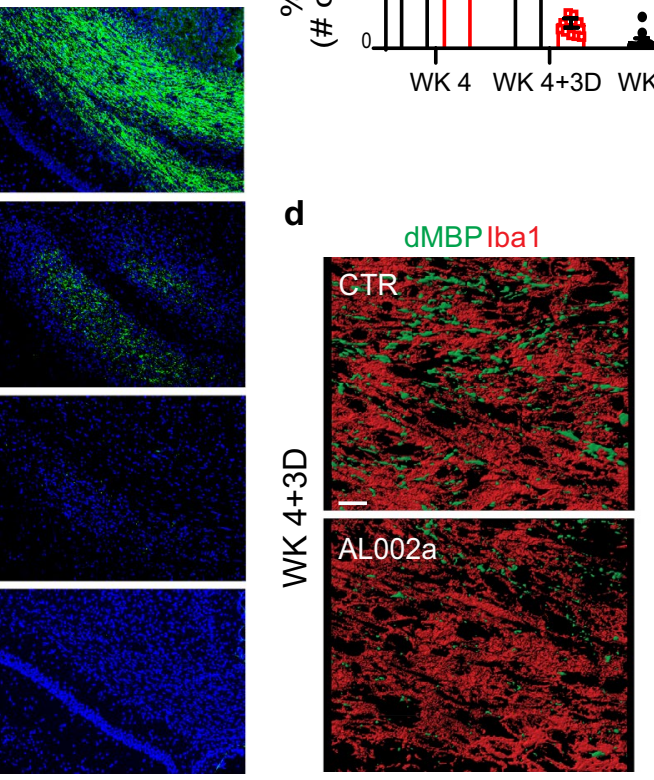

e

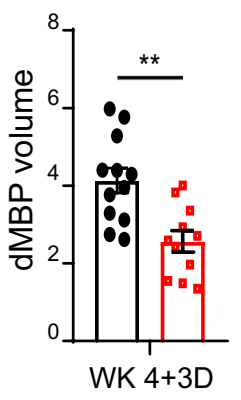

tification of $\mathrm{dMBP}$ debris at the different time points. CTR group: WK $4 N=5$ mice, $n=10$ fields; WK $4+3 \mathrm{D} N=6, n=12$; WK $4+7 \mathrm{D}$ $N=5, n=10$; WK $4+14 \mathrm{D} N=5, n=5 ;$ AL002a-treated group: WK $4 N=5, n=10$; WK $4+3 \mathrm{D} N=5, n=10$; WK $4+7 \mathrm{D} N=4$, $n=8$; WK $4+14 \mathrm{D} N=6, n=6$. $* * * * P<0.0001$, two-tailed unpaired Student's $t$ test. d 3D reconstruction by Imaris of Iba ${ }^{+}$cells (red) and $\mathrm{dMBP}$ (green) immunostaining in the $\mathrm{CC}$ at $\mathrm{WK} 4+3 \mathrm{D}$. Original magnification, $\times 63$. Scale bar, $10 \mu \mathrm{m}$. e Quantification of dMBP total volume per field in mice treated with AL002a or CTR. CTR group: $N=6, n=12$; AL002a treated group: $N=6, n=11$. $* * P<0.01$, twotailed unpaired Student's $t$ test

\section{Treatment of $\mathrm{Trem2}^{+/-}$mice with AL002a antibody accelerates myelin debris clearance}

Next, we assessed the potential impact of AL002a on myelin debris clearance during CPZ-induced demyelination in Trem $2^{+/-}$mice. To this end, Trem $2^{+/-}$mice were treated with AL002a or an isotype control given intraperitoneally 4 days before starting CPZ feeding and then weekly throughout the experiment (Fig. 4a). AL002a was able to reach the brain and it was detected in the CC and in the cortex (Ctx) (Supplementary Fig. 1d). Analyses were performed at 4 weeks (WK 4; when the peak of demyelination is observed) after CPZ feeding and then at 3 days (WK 4+3D), 7 days (WK 4+7D), and 14 days (WK 4+14D) after CPZ withdrawal, when mice 
were fed regular chow to allow remyelination. Treatment in vivo with AL002a resulted in the amelioration of CPZinduced pathology in Trem $2^{+/-}$mice. Specifically, AL002a treatment enhanced myelin debris clearance compared to Trem $2^{+/-}$mice treated with the control antibody, as demonstrated by a significant reduction in $\mathrm{dMBP}$ staining at WK $4+3 \mathrm{D}$ after CPZ withdrawal (Fig. 4b, c). This finding was also confirmed by confocal microscopy and 3D-reconstruction, which showed a significant reduction of dMBP amount (quantified as dMBP volume) per field at the same timepoint in AL002a versus control-treated Trem $2^{+/-}$mice (Fig. 4d, e). Since TREM2 receptor is specifically expressed by microglia in the brain, these results strongly suggest that AL002a antibody accelerates myelin clearance in the CPZ model of demyelination by potentiating TREM 2 receptor function on microglial cells.

\section{AL002a antibody promotes myelin uptake and degradation in vivo and in vitro}

Microglia play a key role in myelin debris removal after CNS demyelination [33]. Microglia internalize and phagocytose myelin which is then degraded intracellularly by lysosomal structures. We hypothesized that reduction in $\mathrm{dMBP}$ staining observed after AL002a treatment could be related to an increased ability to uptake and/or to degrade myelin fragments by $\mathrm{Trem} 2^{+/-}$microglia. To clarify these aspects, the amount of dMBP within microglial phagolysosomes was analyzed by immunofluorescence in the CC at WK 4 and WK 4+3D. Quantitative confocal analysis and 3D reconstruction of $\mathrm{Iba}^{+}$cells showed a significant increase in dMBP amount inside $\mathrm{CD}^{+} 8^{+}$phagolysosomal structures at WK 4 in AL002a vs. control treated mice (Fig. 5a, c). Interestingly, 3 days after toxin withdrawal we observed that dMBP in phagolysosomes was lower in AL002a-treated mice compared to control (Fig. 5b, c; $P=0.07$ ). These findings suggest that AL002a antibody treatment enhanced myelin debris internalization by microglia at WK 4 , as demonstrated by a higher dMBP amount detected inside $\mathrm{CD}^{+} 8^{+}$structures in microglia (Fig. 5a, c), accompanied by decreased dMBP total volume per field (Fig. 4b, c) at WK $4+3 \mathrm{D}$. Moreover, the reduction in $\mathrm{dMBP}$ content within lysosomal structures after 3 days from toxin withdrawal implies that AL002a further accelerated intracellular myelin processing and degradation.

To confirm that AL002a antibody was improving myelin uptake and degradation capacity by microglia, we performed an in vitro myelin phagocytic and degradation assay. To assess myelin phagocytosis, BMDMs from Trem2 ${ }^{+/-}$mice were pre-treated with AL002a or control antibody and then myelin was added to the culture. Analysis of MBP internalization by immunofluorescence revealed a significant increase in MBP amount detected inside $\mathrm{Iba} 1^{+}$cells treated with AL002a after $1 \mathrm{~h}$ (Fig. 5d, e), while no differences between the two groups were detected at 30 ' and $3 \mathrm{~h}$. This suggests a time-dependent increase in myelin uptake by BMDM after AL002a treatment and further supports our in vivo findings showing higher dMBP amount inside microglia at WK 4 on CPZ in AL002 treated group (Fig. 5a, c).

To assess the effect of AL002a on myelin degradation, Trem2 ${ }^{+/-}$BMDM were pre-treated with AL002 or control antibodies, then incubated with human myelin for $2 \mathrm{~h}$, and subsequently washed in order to remove extracellular myelin not taken up. MBP content by BMDM was then quantified after 1, 24, and $48 \mathrm{~h}$. We observed a significant reduction in myelin content in BMDM treated with AL002a compared to those treated with the control antibody both at 24 and $48 \mathrm{~h}$ (Fig. 5f, g). Therefore, AL002a leads to a significant increase in myelin phagocytosis by Trem $2^{+/-}$BMDM after $1 \mathrm{~h}$, and also it accelerates their degradative capacity at later time points. These findings are consistent with the observation that AL002a treatment promotes a more efficient clearance of myelin debris by Trem $2^{+/-}$microglia in vivo in the CPZ model.

\section{Treatment with AL002a enhances the activation state of Trem2 ${ }^{+/-}$microglia}

AL002a could potentially promote myelin debris clearance by Trem $^{+/-}$microglia after CPZ by increasing microglia numbers in the areas of demyelination and/or their phagocytic functions. Since we did not observe any difference in microglia density (Supplementary Fig. 2a and b) nor proliferation (the latter quantified as $\mathrm{Iba} 1^{+} \mathrm{BrdU}^{+}$cells at WK $4+3 \mathrm{D}$; Supplementary Fig. $2 \mathrm{c}$ and d) in Trem $2^{+/-}$mice after treatment with AL002a, we hypothesized that AL002a antibody could enhance microglia activation state as well as phagocytic and lysosomal activity.

FACS analysis of CD11 $\mathrm{b}^{+} \mathrm{CD} 45^{\text {int }}$ microglia isolated from the CC (Fig. 6a and Supplementary Fig. 3a) showed a significant increase in CD80 costimulatory molecule at WK 4 measured both as mean fluorescence intensity (MFI) and as cell percentage in AL002a treated vs. control (Fig. 6b, c). No differences were observed in CD86 and CD11b expression on microglia (Fig. 6b, c). Iba1 intensity in the CC, detected by immunofluorescence, was also higher in AL002a-treated compared to the control group at WK 4 (Fig. 6f, g), thus confirming the increased activation of microglia after AL002a treatment. At 3 days after $\mathrm{CPZ}$ withdrawal, there were no differences in microglia activation state between the two groups (Fig. 6c-e), thus indicating that $\mathrm{CPZ}$ removal was sufficient to revert AL002a effects on microglia inflammatory state. A strong upregulation of activation markers after AL002a treatment was also detectable in microglia isolated from the hippocampus (HP) at WK 4 (Supplementary Fig. $3 \mathrm{~b}-\mathrm{g}$ ), an area which is also affected by pathology after 
c

a
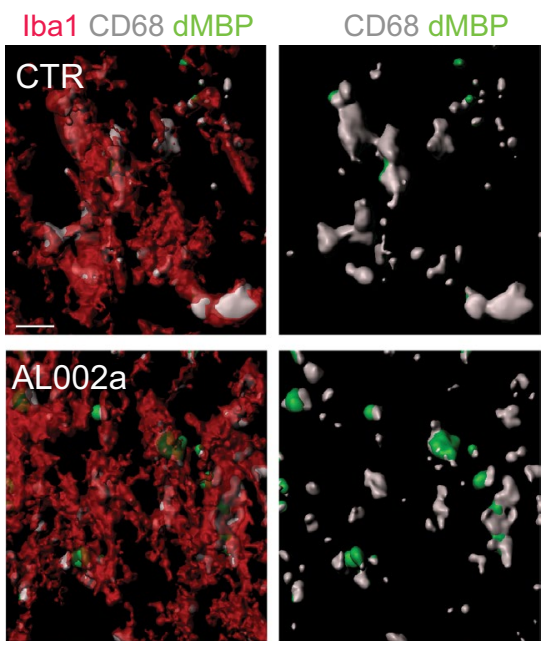

d
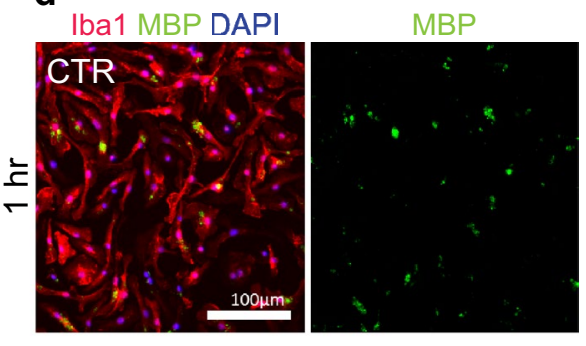

$\mathbf{f}$
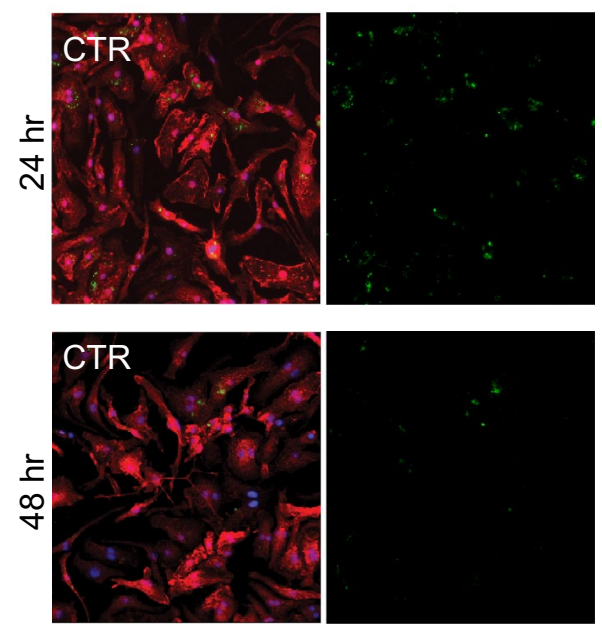

b
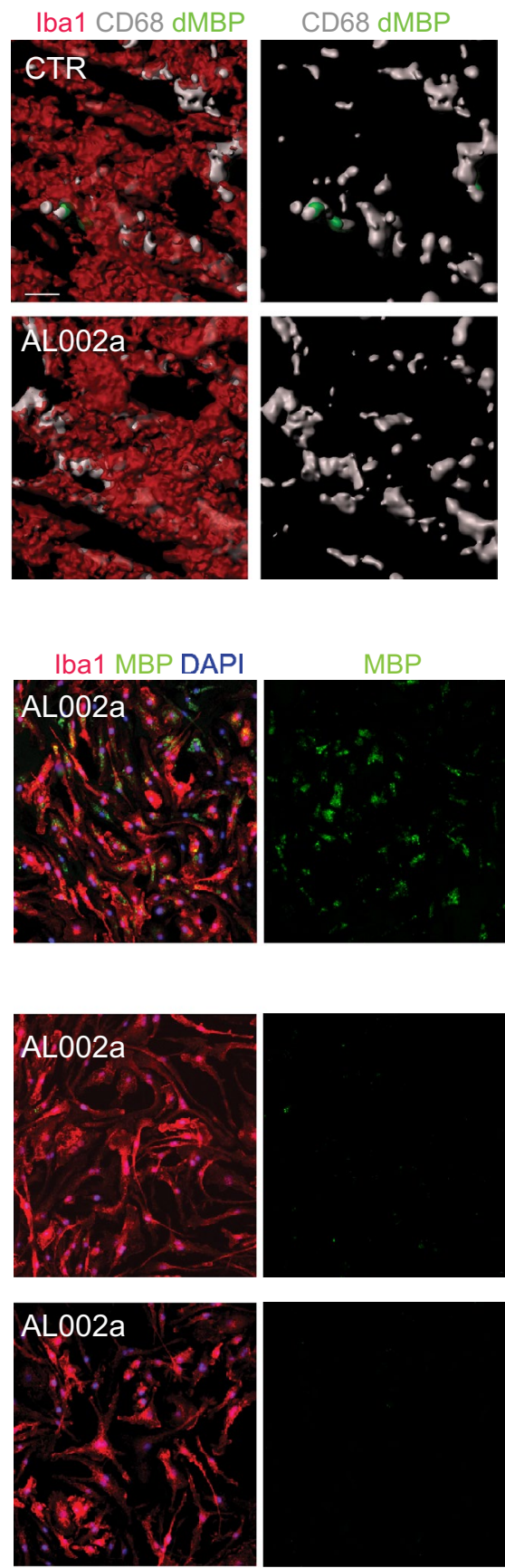

CD68 dMBP
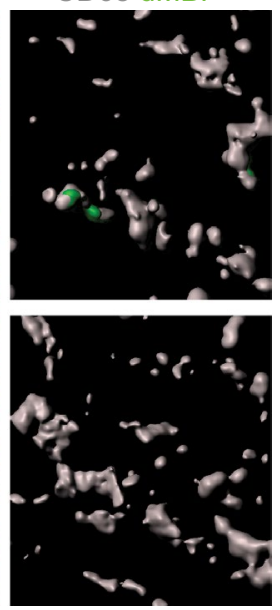
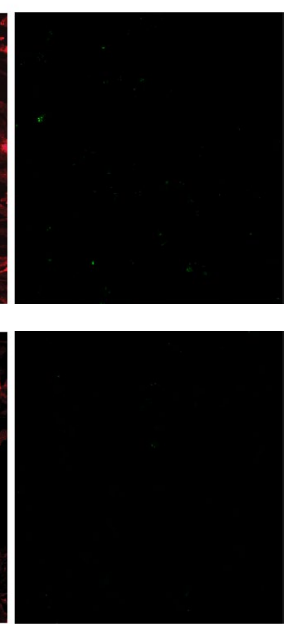

\section{e}
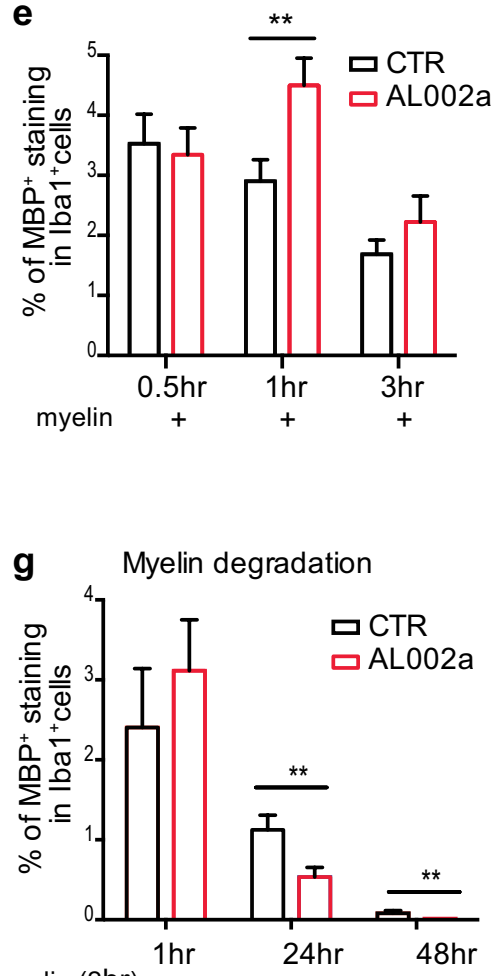

myelin (2hr)

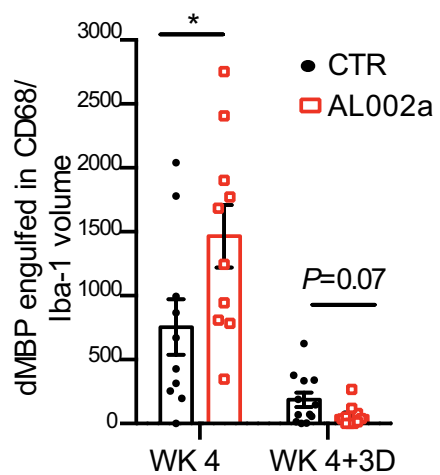

Myelin phagocytosis
Fig. 5 AL002a treatment enhances myelin uptake and processing by Trem $^{+/-}$microglia in vivo and by BMDM in vitro. Representative confocal $\mathrm{z}$-stack and relative $3 \mathrm{D}$ surface rendering showing volume reconstruction of microglia (red), CD68 (gray) and dMBP (green), detected within microglial $\mathrm{CD}^{+} 8^{+}$structures at WK 4 (a) and WK $4+3 \mathrm{D}$ (b) in the CC of Trem $2^{+/-}$mice. Original magnification, $\times 63$. Scale bar, $3 \mu \mathrm{m}$. c Quantification of engulfed dMBP within CD68 per microglia at WK 4 and WK $4+3 \mathrm{D}$. CTR group: WK $4 N=5$ mice, $n=10$ fields; WK $4+3 \mathrm{D} N=6, n=12$. AL002a-treated group: WK $4 N=5, n=10$; WK $4+3 \mathrm{D} N=6, n=11 ; * P<0.05$, twotailed unpaired Student's $t$ test. d Representative images and e relative quantification of $T r e m 2^{+/-}$BMDM pre-stimulated in vitro with AL002a and control antibodies $(10 \mu \mathrm{g} / \mathrm{ml})$ and treated with myelin for $0.5,1$ and $3 \mathrm{~h}$. Iba1 (red), MBP (green), DAPI (blue). Original magnification, $\times 10$. Scale bar, $100 \mu \mathrm{m}$. CTR group: 30' $n=80$; $1 \mathrm{~h} n=100 ; 3 \mathrm{~h} n=80$; AL002a group: 30' $n=80 ; 1 \mathrm{~h} n=100 ; 3 \mathrm{~h}$ $n=80$. $* * P<0.01$, two-tailed unpaired Student's $t$ test. f Representative images and $\mathbf{g}$ relative quantification of Trem $2^{+/}$BMDM assayed for their degradative capacity. Trem $2^{+/-}$BMDM were pre-stimulated with AL002a or control antibodies $(10 \mu \mathrm{g} / \mathrm{ml})$, then incubated with human myelin for $2 \mathrm{~h}$ and washed. MBP content in $\mathrm{Iba}^{+}$cells was quantified after 1, 24, and $48 \mathrm{~h}$. CTR group: $1 \mathrm{~h} n=42 ; 24 \mathrm{~h} n=104$; $48 \mathrm{~h} n=80$; AL002a group: $1 \mathrm{~h} n=48 ; 24 \mathrm{~h} n=110 ; 48 \mathrm{~h} n=80$. $* * P<0.01$, two-tailed unpaired Student's $t$ test. d-g Three independent experiments were performed 
a
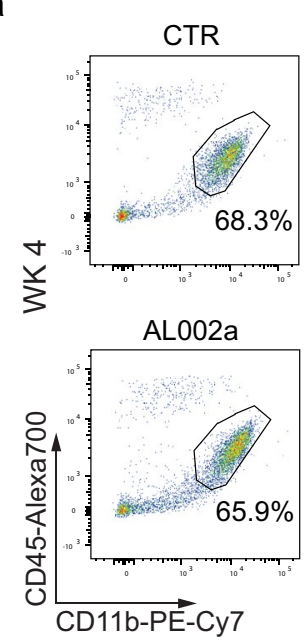

b $\square$ CTR

口ALO02a
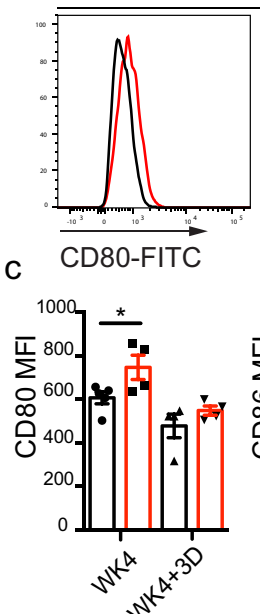

|ba1 DAPI

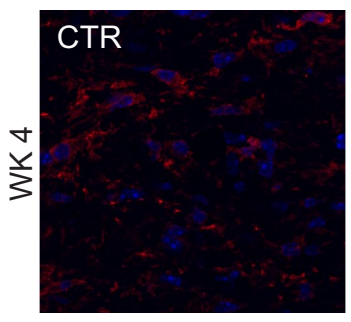

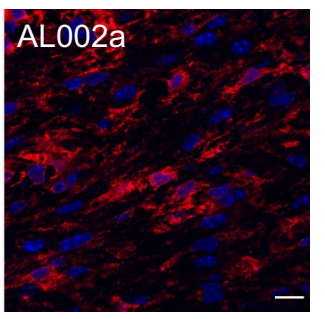

WK 4
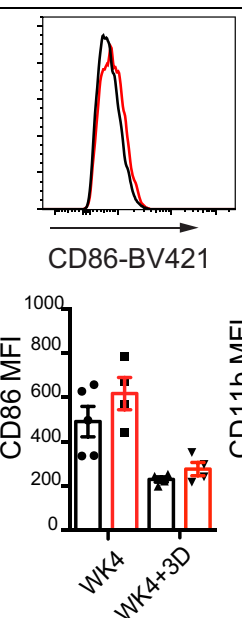

d
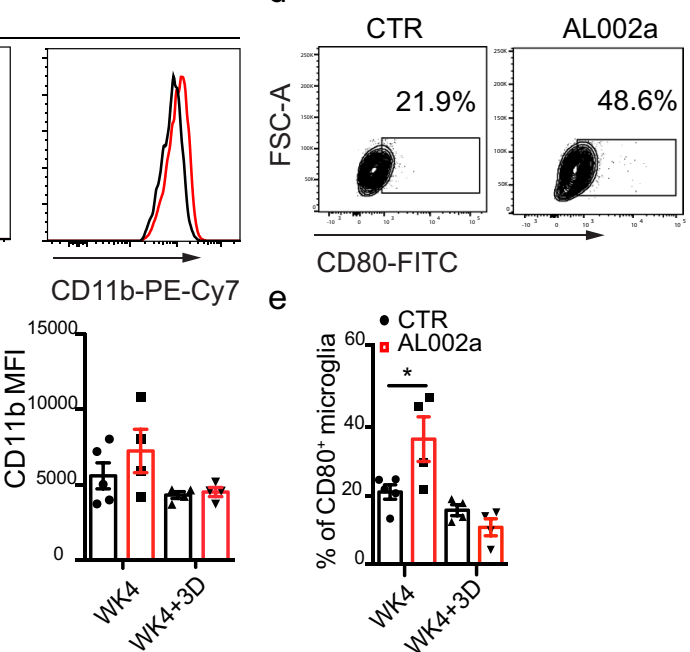

g

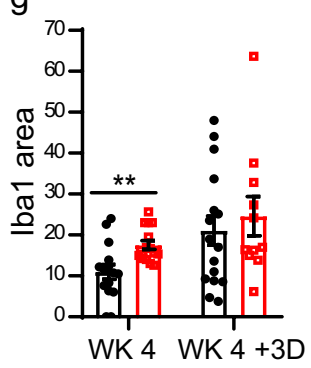

$\mathrm{h}$
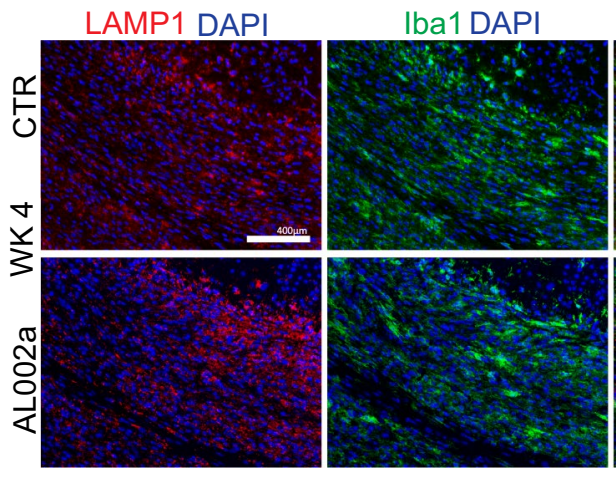

LAMP1 |ba1 DAPI

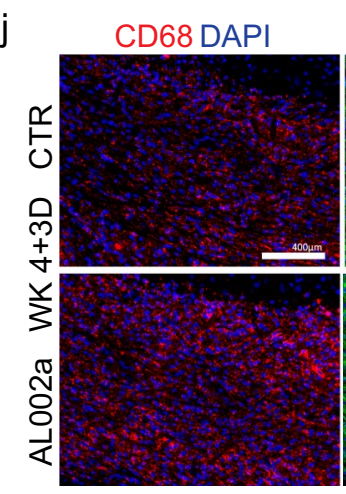

|ba1 DAP|
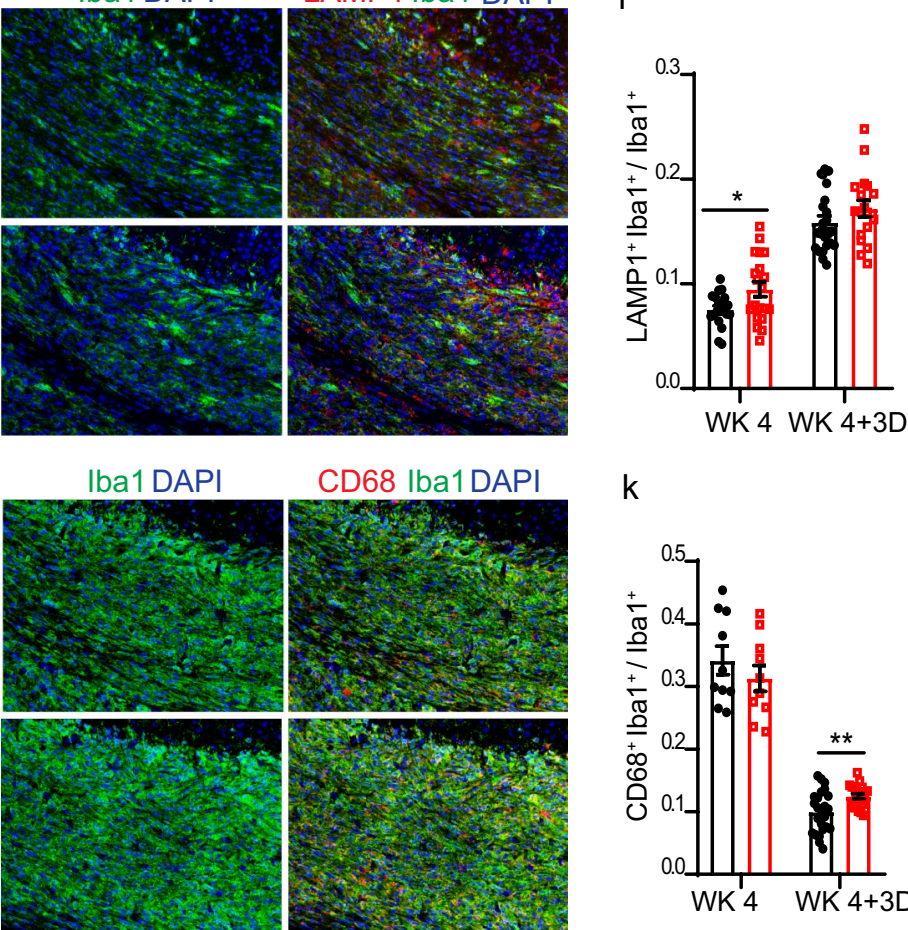

k

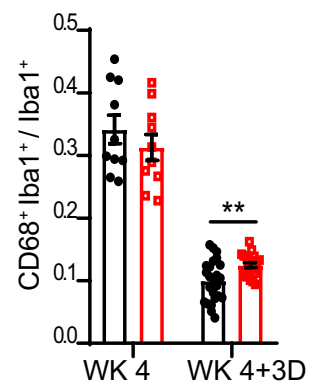

CPZ administration [15]. This result further corroborates AL002a effect in promoting microglia activation in the CPZ model of demyelination.
Additionally, at WK 4 after CPZ, the lysosomal-associated membrane protein 1 (LAMP1), a protein essential for the fusion of phagosomes with lysosomes and their 
4Fig. 6 Increased Trem2 ${ }^{+/-}$microglia activation following AL002a antibody treatment in vivo. a FACS analysis of $\mathrm{Trem}^{+/-}$microglia isolated from the $\mathrm{CC}$ at WK 4. Microglia were identified as $\mathrm{CD} 11 \mathrm{~b}^{+} \mathrm{CD} 45^{\text {int }}$ cells upon doublet discrimination and death cell exclusion. Values indicated in FACS plots represent the relative frequency of live cells after duplets exclusion. b Histograms showing the mean fluorescence intensities (MFIs) of surface CD80, CD86, and $\mathrm{CD} 11 \mathrm{~b}$ at WK 4 and $\mathbf{c}$ their relative quantification at WK 4 and WK 4+3D. d Example plots of flow cytometric analysis of CD80 positive microglia at WK 4 and e quantification of the microglia percentage expressing CD80 molecule at WK 4 and WK $4+3 \mathrm{D}$. CTR group $N=5$ and AL002a treated group $N=4 ; * P<0.05$, two-tailed unpaired Student's $t$ test. f Representative confocal images of Iba1 (red) and DAPI (blue) in the CC at WK 4. Original magnification, $\times 63$. Scale bar, $10 \mu \mathrm{m}$. g Quantification of Iba1 intensity (measured as area) at WK 4 and WK $4+3 \mathrm{D}$. CTR group: WK $4 N=5$ mice, $n=15$ fields; WK $4+3 \mathrm{D} N=6, n=16$; AL002a-treated group: WK $4 N=5, n=15$; WK $4+3 \mathrm{D} N=6, n=11 ; * * P<0.01$ two-tailed unpaired Student's $t$ test. h Representative images of LAMP1 (red), Iba1 (green) and DAPI (blue) at WK 4. Original magnification, $\times 20$. Scale bar, $400 \mu \mathrm{m}$. i Quantification of LAMP1 ${ }^{+}$signal colocalizing with Iba $1^{+}$cells. CTR group: WK $4 N=5, n=20$; WK $4+3 \mathrm{D} N=6$, $n=23$; AL002a treated group: WK $4 N=5, n=20$; WK $4+3 \mathrm{D} N=5$, $n=18 ; * P<0.05$, two-tailed unpaired Student's $t$ test. j Representative images of CD68 (red), Ibal (green) and DAPI (blue) at WK 4. Original magnification, $\times 20$. Scale bar, $400 \mu \mathrm{m}$. k Quantification of $\mathrm{CD}^{+} 8^{+}$signal colocalizing with $\mathrm{Iba}^{+}$cells. CTR group: WK $4 N=5$, $n=10$; WK 4+3D $N=6, n=24$; AL002a-treated group: WK $4 N=5$, $n=10$; WK $4+3 \mathrm{D} N=5, n=20 ; * * P<0.01$ two-tailed unpaired Student's $t$ test

subsequent degradation [12], was significantly increased in microglia from AL002a-treated mice compared to controls (Fig. 6h, i). WK 4 represents the peak of demyelination and microglia are actively phagocytosing myelin debris at this time. Notably, we also detected a significant increase in $\mathrm{CD} 68^{+}$phagosome structures in Trem $2^{+/-}$microglia at WK 4+3D compared to control (Fig. 6j, k). This further supports enhanced in vivo phagocytic activities by microglia following AL002a-induced activation.

\section{AL002a treatment leads to increased OPC density, OL maturation, and remyelination}

Microglia activation and myelin debris clearance are critical steps for successful remyelination [29]. Therefore, we hypothesized that AL002a treatment, by promoting myelin debris clearance by microglia, could positively impact remyelination. We have previously shown that increased accumulation of damaged myelin in the $\mathrm{CC}$ of Trem $2^{+/-}$mice, compared to Trem $2^{+/+}$mice, was associated with a reduced number of PDGFR $\alpha^{+}$OPCs (Fig. 3a, b, d, e). Efficient clearance of myelin debris after demyelination can favorably impact OPC recruitment and differentiation into remyelinating oligodendrocytes [29]. We observed a significant increase in PDGFR $\alpha^{+}$OPC density in the $\mathrm{CC}$ of Trem2 ${ }^{+/-}$mice after AL002a antibody treatment at WK $4+3 \mathrm{D}$ and WK 4+7D compared to control (Fig. 7a, b).
No differences in PDGFR $\alpha^{+}$OPC proliferation, quantified as density of PDGFR $\alpha^{+} \mathrm{BrdU}^{+}$cells, were observed at WK 4+3D (Supplementary Fig. 4a, b). These results suggest that AL002a antibody could promote the recruitment of OPCs from surrounding brain regions, rather than enhancing the proliferation of resident OPCs in the $\mathrm{CC}$. We then analyzed the expression of OLIG2 and CNPase, markers specifically expressed by mature OL. We observed a significant increase of both OLIG2 ${ }^{+}$and $\mathrm{CNPase}^{+}$cells upon AL002a treatment vs. control at WK $4+3 \mathrm{D}$ after $\mathrm{CPZ}$ withdrawal (Fig. 7c-e). The same increase in Olig2 and Cnp genes was detected by RT-qPCR analyses at WK $4+7 \mathrm{D}$ in both the $\mathrm{CC}$ and HP (Supplementary Fig. 4c and d). In parallel, we observed a significant increase in the mRNA of myelin genes such as myelin basic protein $(M b p)$, myelin-associated protein $(M a g)$, and proteolipid protein $1(P l p l)$ in both regions (Fig. 7f-h). Finally, to assess remyelination and thickness of the new myelin sheaths in the $\mathrm{CC}$, we performed transmission electron microscopy (TEM) analysis on sagittal sections of Trem $2^{+/-}$mice naïve or fed with CPZ and analyzed after withdrawal at WK 4+3D and WK 4+7D (Fig. 7i). Specifically, myelinated axons (MA) and naked axons were counted in the CC of naïve mice (MA: $89 \% \pm 0.00724$ as mean \pm SEM) or in mice fed with $\mathrm{CPZ}$ and treated with AL002a or control antibody. Significantly more MA were counted in the CC after AL002a treatment compared to control at WK 4+3D (MA: 32\% 0.0192 in AL002a-treated group vs. MA: $23 \% \pm 0.0122$ in control group) and this was even more pronounced at WK 4+7D (MA: 53\% 0.0124 in AL002a-treated group vs. MA:42\% \pm 0.0179 in control group) (Fig. 7j). In addition, g-ratio analysis revealed significantly increased myelin sheath thickness in mice treated with AL002a at WK 4 +3D and WK 4 + 7D compared to control (Fig. 7k). All together, these data strongly suggest that activation of TREM2, through AL002a antibody, increased OPC accumulation within the $\mathrm{CC}$ lesioned area, enhanced OPC differentiation into mature oligodendrocyte, and accelerated remyelination.

\section{AL002a treatment preserves axonal integrity}

Remyelination is known to protect axons from ongoing degeneration [18]. Therefore, we further investigated whether AL002a together with promoting remyelination was also positively impacting axonal health. We assessed levels of neurofilament-light chain (Nf-L), which are released in significant quantity following axonal damage or neuronal degeneration, in Trem $2^{+/-}$mice treated with AL002a or control antibody. Mice treated with AL002a showed significantly lower Nf-L levels in the plasma at WK 4+3D compared to the control, with a trend of reduced levels also observed at WK $4+7 \mathrm{D}$ (Fig. 8a). These results are suggesting that AL002a treatment during CPZ-induced CNS 
a
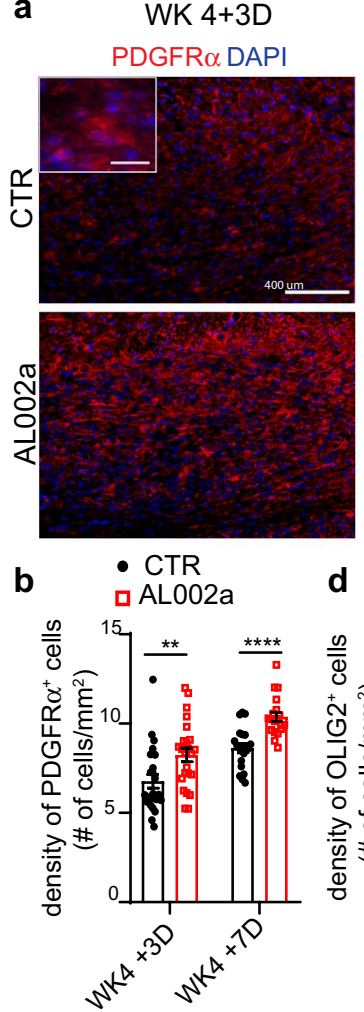

i
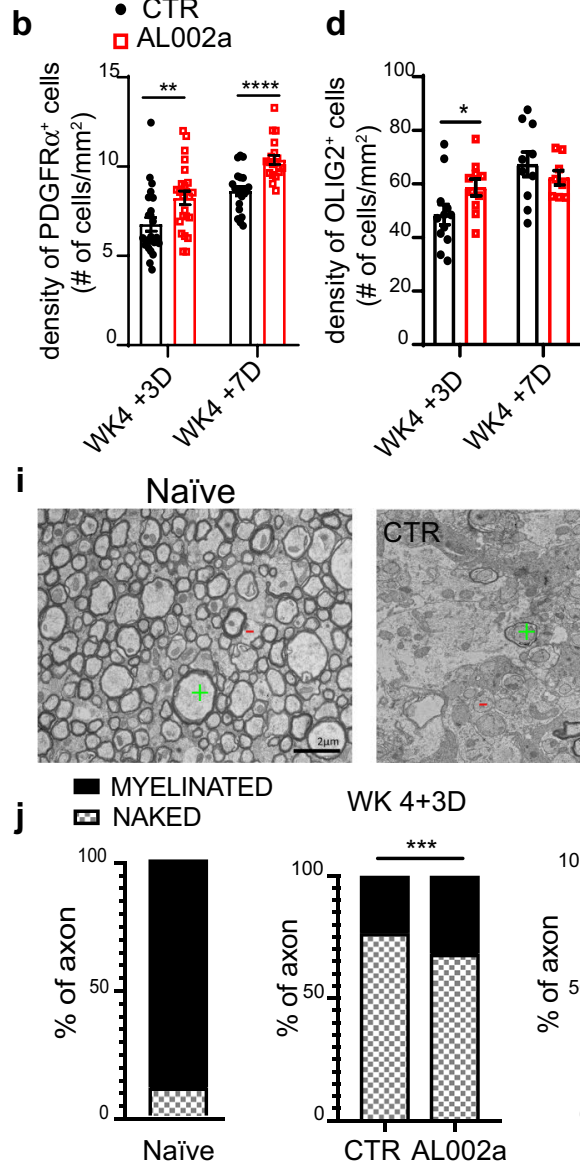

e

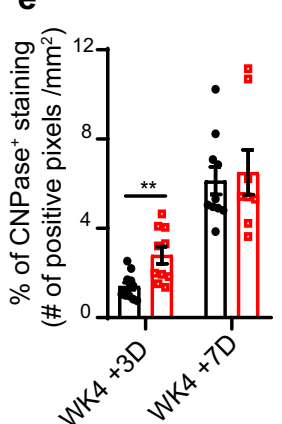

WK 4+3D
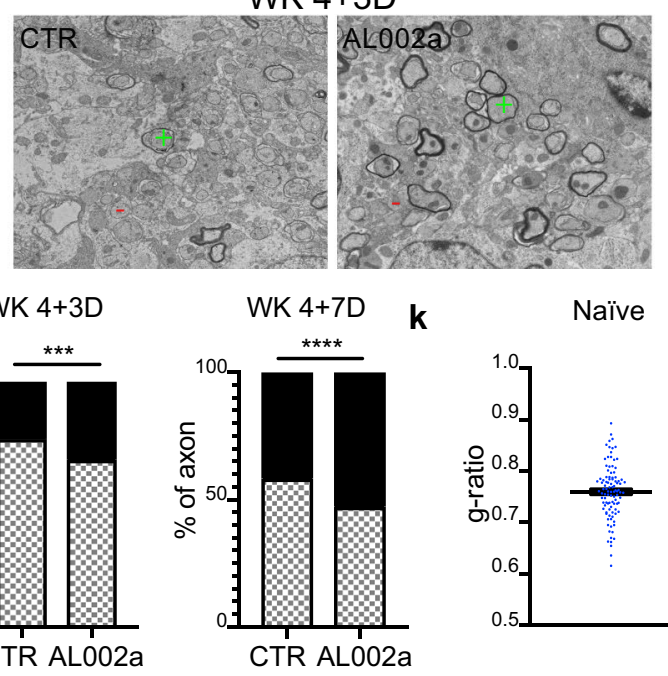

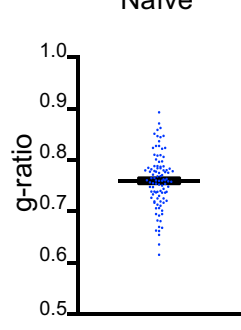

- CTR

- AL002a

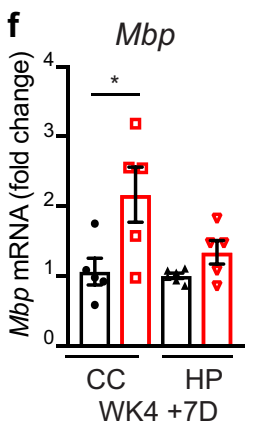

C
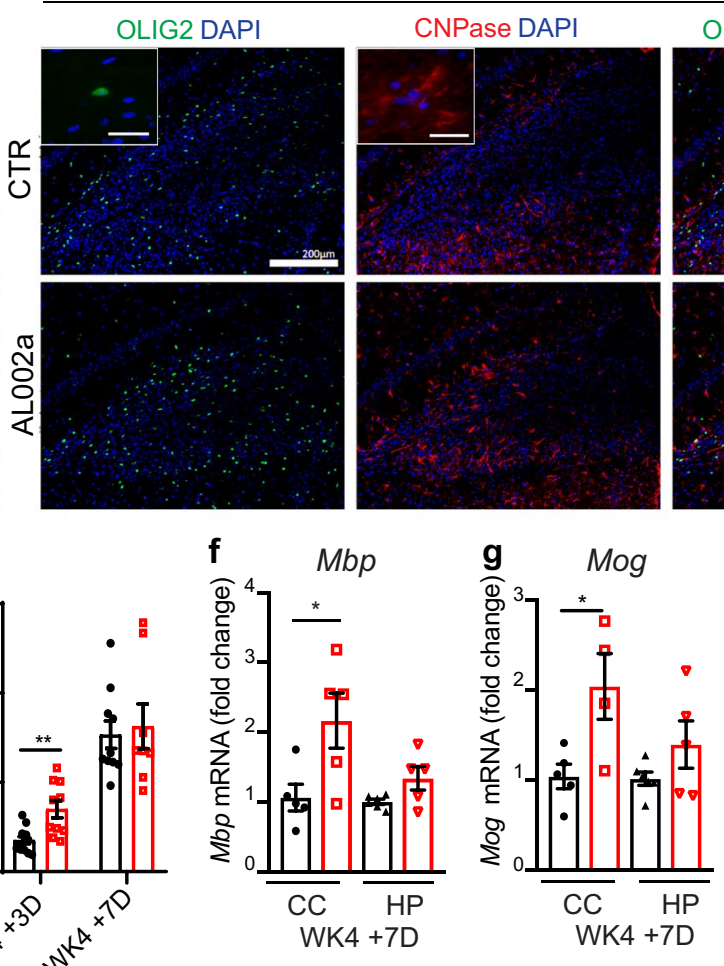

OLIG2 CNPase DAPI
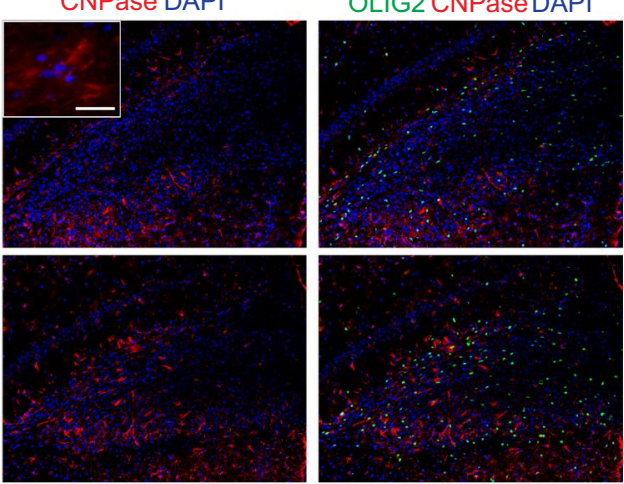

h Plp1

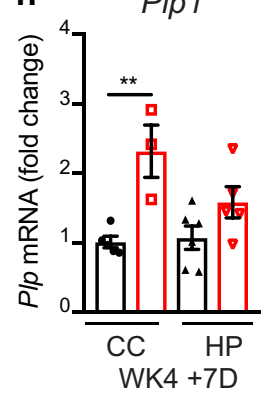

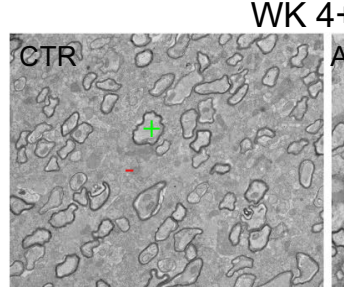

WK $4+3 D$
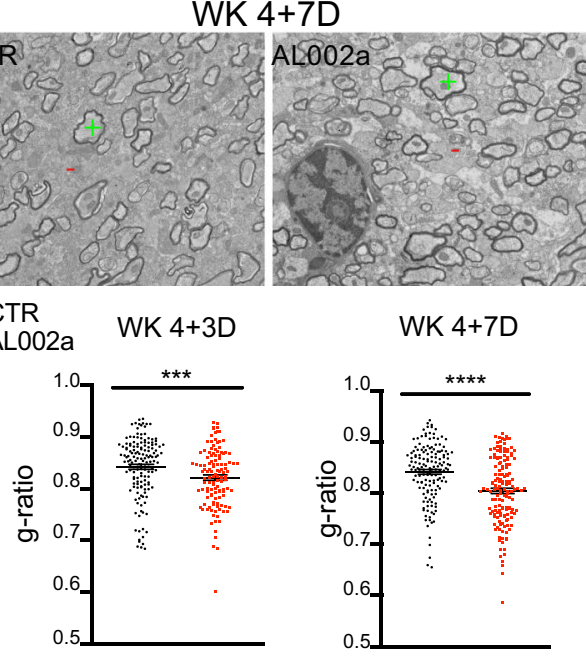

demyelination will also preserve axonal integrity. Further evidence supporting a neuroprotective effect exerted by AL002a, came from the evaluation with the SMI-31 antibody (a marker of healthy axons) in the $\mathrm{CC}$, which was significantly increased in AL002a compared to control treated Trem $2^{+/-}$mice at WK $4+7 \mathrm{D}$ (Fig. 8b, c). In conclusion, AL002a treatment preserved axonal health during CPZ treatment, thus preventing neurodegeneration.

\section{Macrophages derived from Nasu-Hakola subjects display a defect in phagocytic pathways}

TREM2 function in human microglia is still elusive and this is also due to experimental difficulties and limitations of human studies. A role of TREM2 in phagocytic pathways in the human brain was previously suggested [13]. Here, we have analyzed the gene expression profile of macrophages obtained from the peripheral blood of three patients affected by NHD (lacking TREM2 receptor), compared to healthy control individuals. NHD is characterized by loss of myelin 
४Fig.7 AL002a -treated mice show increased OPC density, mature myelin and remyelination after CPZ-induced demyelination. a Representative images of PDGFRo $\alpha^{+}$(red) and DAPI (blue) in the CC at WK 4+3D and WK 4+7D. Original magnification, $\times 20$. Scale bar, $400 \mu \mathrm{m}$. Insert magnification, $\times 60$. Scale bar, $25 \mu \mathrm{m}$. b Quantification of PDGFR $\alpha^{+}$cell density. CTR group: WK $4+3 \mathrm{D} N=6$ mice, $n=24$ fields; WK $4+7 \mathrm{D} N=5, n=20$; AL002a-treated group: WK $4+3 \mathrm{D} N=6, n=24$; WK $4+7 \mathrm{D} N=5, n=20 ; * * P \leq 0.01$; $* * * * P \leq 0.0001$; WK $4+3 \mathrm{D}$ two-tailed Mann-Whitney test; WK 4+7D two-tailed unpaired Student's $t$ test. c Representative images of OLIG2 (green), CNPase (red) and DAPI (blue) in the CC at WK 4+3Ds and WK 4+7D. Original magnification, $\times 10$. Scale bar, $200 \mu \mathrm{m}$. Insert magnification, $\times 60$. Scale bar, $25 \mu \mathrm{m}$. d Quantification of the density of $\mathrm{OLIG}_{2}{ }^{+}$cells at both the time points. $\mathrm{e}$ Quantification of the percentage of $\mathrm{CNPase}^{+}$staining in the CC. CTR group: WK 4+3D $N=6, n=12$; WK $4+7 \mathrm{D} N=5, n=10$; AL002a-treated group: WK 4+3D $N=5, n=10$; WK 4+7D $N=4$, $n=8 ; * P<0.05$, ${ }^{*} P \leq 0.01$; two-tailed unpaired Student's $t$ test. RTqPCR quantification of mRNA levels of myelin basic protein $(\mathrm{Mbp})$ (f), myelin oligodendrocyte glycoprotein $(\mathrm{Mog})(\mathrm{g})$ and proteolipid protein 1 (Plp1) (h) in the corpus callosum (CC) and the hippocampus (HP) of Trem $2^{+/-}$mice treated with AL002a antibody or CTR. Tissues were collected at WK 4+7D. CTR group: $N=5$, AL002atreated group: $N=5(M p b) ; N=4(M o g) ; N=3(P l p l) . * P<0.05$, two-tailed unpaired Student's $t$ test. i Electron microscopy (EM) of a naïve mouse and mice treated with AL002a or isotype control antibody at WK $4+3 \mathrm{D}$ and $\mathrm{WK} 4+7 \mathrm{D}$. Green plus sign indicates myelinated axons and red minus sign indicates naked axons. Original image $=\times 5000$. Scale $b a r=2 \mu \mathrm{m}$. j Percentage of myelinated axons (MA) and naked axons (NA) in naïve mice, and in CTR and AL200a-treated mice at WK $4+3 \mathrm{D}$ and $\mathrm{WK} 4+7 \mathrm{D}$. $* * * P<0.001$ at WK $4+3 \mathrm{D}$ and $* * * * P<0.0001$ at WK $4+7 \mathrm{D}$, two-tailed MannWhitney test. $\mathbf{k}$ G-ratio of myelinated fibers in naïve mice, and in CTR and AL002a-treated mice at WK 4+3D and WK 4+7D. Each dot represents one axon. Naïve mice $N=2$, CTR treated mice at WK $4+3 \mathrm{D} N=5$ and at WK $4+7 \mathrm{D} N=5$; AL002a-treated mice at WK $4+3 \mathrm{D} N=4$ and at WK $4+7 \mathrm{D} N=5$. $* * * P<0.001$ at WK $4+3 \mathrm{D}$ and $* * * * P<0.0001$ at WK $4+7 \mathrm{D}$, two-tailed unpaired Student's $t$ test

and microglia activation in the CNS [25]. These studies aimed to investigate whether a TREM2 loss of function mutation in NHD patients could result in the dysregulation of gene expression pathways involved in the clearance of myelin debris in the brain.

The cohort of subjects that we studied was composed of: (1) two siblings affected by NHD and carrying an homozygous loss-of-function point mutation in exon 2 of the TREM2 gene (C97T) causing a glutamine in position 33 to be changed in a stop codon (Q33X) [4]; (2) one unaffected sibling from the same family, homozygous for the normal TREM2 allele, who was included as a control; (3) one NHD subject carrying a different homozygous mutation in the splice-donor consensus site of intron 3 of TREM2 $(482+2 \mathrm{~T} \rightarrow \mathrm{C})$ [9]; (4) two unrelated healthy control subjects. Genetic and demographic characteristics are summarized in Table 2. Macrophages from each subject were differentiated in vitro from peripheral blood monocytes, and gene expression analysis was performed by microarray analysis. A total of 20,874 genes were tested to compare their expression between NHD subjects $(n=3)$ and unaffected controls $(n=3)$ ) 2526 genes were found to be differentially expressed with absolute fold change $>2$ (ANOVA $P$ value $<0.05)$. The heat map for these genes is displayed in Fig. 9a. Only 11 genes, including TREM2, remained significant after adjustment of the absolute fold change cutoff to a FDR < 0.05 [40] (Supplementary Table 1). Principal component analysis showed the ability of gene expression data to discriminate NHD from control subjects (Supplementary Fig. 4e). Gene ontology (GO) analysis revealed that genes involved in the phagosome pathway are among the toppathways significantly altered in NHD subjects compared to controls along with changes in immune response pathways (Fig. 9b, and Supplementary Table 2 and 3). Interestingly, differentially expressed genes in the phagosome pathway were all downregulated in NHD vs. unaffected control subjects. The distribution of their fold changes and $P$ values is represented in a volcano plot in Fig. 9c. Furthermore, we performed a co-expression analysis for TREM2 using public RNA-seq data from 31,499 samples and then compared these genes with the differentially expressed genes in NHD patients (Supplementary Table 4). A GO cellular component analysis confirmed that genes from the phagocytic pathway are both significantly co-expressed with TREM 2 and altered in NHD patients (Supplementary Table 5). Overall, these data from NHD individuals, confirmed that TREM2 receptor plays a critical role in phagocytic pathways in human myeloid cells and that during NHD pathology genes involved in phagocytosis are specifically dysregulated.

\section{Discussion}

This study provides evidence of beneficial effects of treatment with the new agonistic anti-TREM2 antibody AL002a in the CPZ model of CNS demyelination. We showed that TREM2 is highly expressed on microglia/macrophages phagocyting myelin in MS active lesions. In CPZ-induced demyelination we further validated previous findings that loss of TREM2 reduces myelin clearance and we showed that loss of one copy of TREM2 has an intermediate phenotype, suggesting that myelin removal by microglia is sensitive to TREM2 copy numbers. We then demonstrated that treatment with AL002a in vivo promotes efficient clearance of myelin debris, with increased myelin phagocytosis and intracellular degradation after CPZ-induce demyelination. This was accompanied by increased microglia activation and by a higher expression of proteins involved in phagocytic and degradative pathways. Most importantly, AL002a treatment increased OPC density and differentiation into mature OL to support remyelination, thus finally leading to axonal health preservation. Notably, macrophages from NHD individuals with loss-of-function homozygous TREM2 mutations 
a

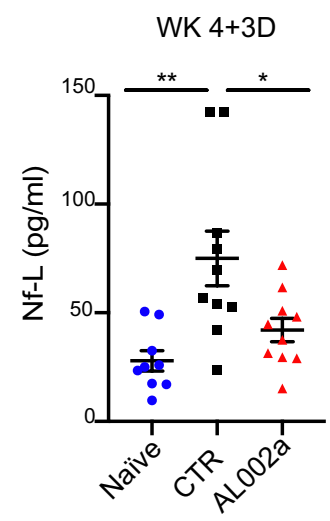

b

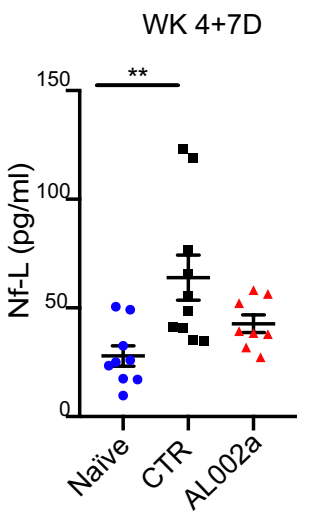

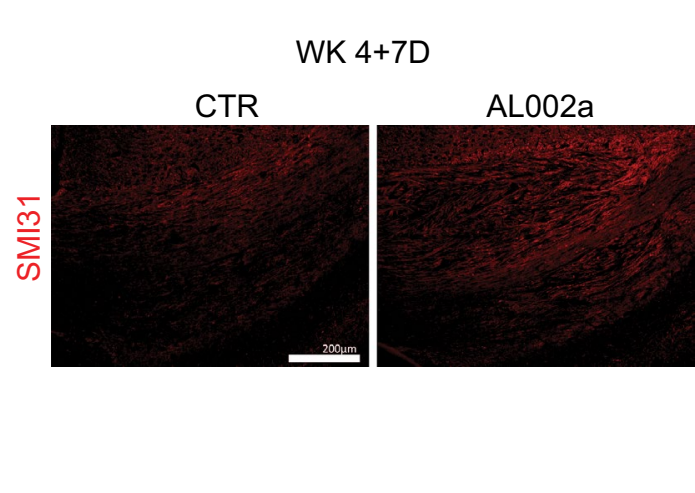

C

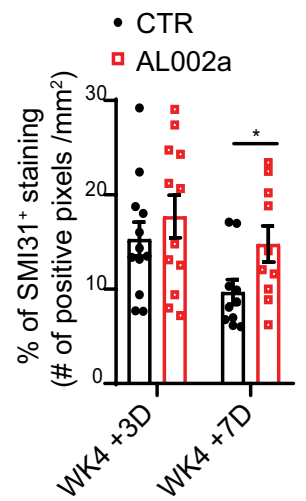

Fig. 8 AL002a positively impacts axonal health after CPZ-induced demyelination. a Neurofilament light chain (Nf-L) levels were measured in plasma samples collected from Trem $2^{+/}$naïve mice and Trem2 ${ }^{+/-}$mice treated with AL002a antibody or CTR at WK $4+3 \mathrm{D}$ and WK $4+7 \mathrm{D}$ after CPZ-induced CNS demyelination. Naïve mice, $N=9$; CTR group: WK 4+3D $N=10$; WK 4+7D $N=10$; AL002atreated group: WK $4+3 \mathrm{D} N=10$; WK $4+7 \mathrm{D} N=8$. $* P<0.05$,

showed a defect in the phagocytic pathways, suggesting that findings from mouse studies may translate to humans.

Growing evidences suggest the important role of TREM2 in microglia function. We previously showed that Trem $2^{-/-}$mice have a striking defect in microglia activation and myelin debris clearance in the CPZ model [8]. Similarly, lack of TREM2 in AD experimental models led to reduced microglia number, clustering and activation around CNS amyloid plaques accompanied by decreased plaque compaction and increased neuritic damage [50, 51, 53]. In line with these observations, TREM2 was demonstrated to mediate the switch from a homeostatic to a disease-associated microglia (DAM) phenotype [21]. TREM2 on microglia may also act as a lipid sensor capable of binding myelin debris with subsequent engulfment and clearance [42]. Our data demonstrate that AL002a is a potent TREM2 agonist antibody capable of activating TREM 2 in vitro and in vivo and to potentiate myelin-induced activation of TREM2 signaling. We further characterized the role of AL002a in the in vivo model of CPZ-induced CNS demyelination. First, we tested AL002a in Trem $2^{+/+}$mice, without detecting any difference in damaged myelin accumulation, number of microglia or OPC in the CC compared to the control group at 4 weeks on CPZ (data not shown). These results were not unexpected given the rapid microglia response to damage, efficient clearance of myelin debris and subsequent remyelination after CPZ in wild-type mice [17]. Next, we tested AL002a in Trem $2^{+/-}$mice, which had less efficient clearance of damaged myelin compared to their wild-type counterparts, giving us the opportunity to unveil any effect of antibodymediated TREM2 activation on CPZ-induced pathology.
**P $\leq 0.01$; one-way ANOVA with Tukey's post hoc test. b Representative images and $\mathbf{c}$ quantification of SMI31 (red) fluorescent staining at WK $4+3 \mathrm{D}$ and $\mathrm{WK} 4+7 \mathrm{D}$ in the CC. CTR group: WK $4+3 \mathrm{D} N=6$ mice, $n=12$ fields; $4+7 \mathrm{D} N=5, n=10$; AL002a-treated group: WK $4+3 \mathrm{D} N=6, n=12$; WK $4+7 \mathrm{D} N=5, n=10$ fields; $* P<0.05$; two-tailed unpaired Student's $t$ test

Indeed, AL002a treatment in vivo significantly enhanced myelin debris clearance in Trem $2^{+/-}$mice compared to the control Trem $2^{+/}$group. This was likely due to a direct effect of AL002a on microglia, which consistently was shown to express TREM2 [45]. AL002a treatment in vivo by activating TREM2-dependent intracellular pathways led to more efficient myelin phagocytosis and degradation by microglia. This was accompanied by increased microglia expression of markers of activation (Iba1 and CD80) and phagolysosomal activity (CD68 and LAMP1). Similar effects were observed in BMDM in vitro. Our results clearly suggest that TREM2 plays a key role in myelin engulfment and intracellular processing by microglia. TREM 2 has often been referred to as a phagocytic receptor as it was demonstrated to be involved in microglia phagocytosis of apoptotic neurons [46], myelin [8] and $\beta$-amyloid [24]. However, our current and previous findings indicate a more complex TREM 2 function not only in myelin uptake, but also in promoting myelin debris degradation through the phagolysosomal pathway [8]. These findings were corroborated by the analysis performed in macrophages derived in vitro from NHD patients revealing that genes involved in the phagosome pathway are significantly altered in NHD subjects compared to healthy controls along with changes in immune response pathways. Therefore, myelin and axonal degeneration observed in NHD underscore a critical function played by microglia and TREM 2 in myelin clearance, phagocytic functions, and maintenance of neuronal integrity.

We have previously reported that Trem $2^{-1-}$ mice have a significant defect compared to Trem $2^{+/+}$in microglia numbers and proliferation at 4 weeks on CPZ and this could 
a

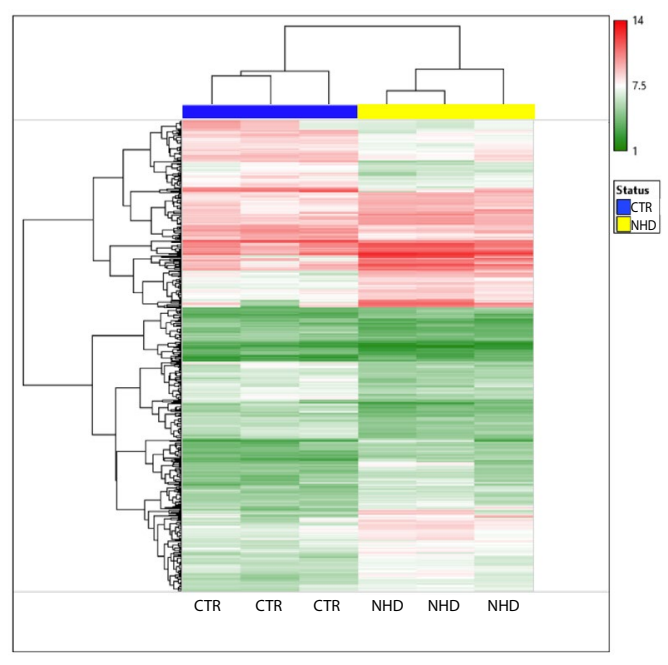

C

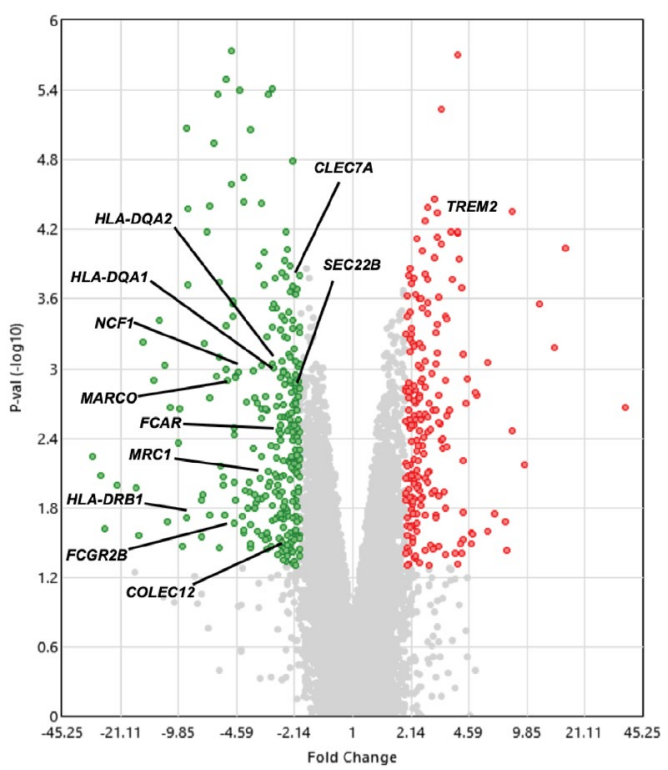

Fig. 9 Phagocytic pathways dysregulation in macrophages derived from individuals affected by Nasu-Hakola disease. a Heat map showing different expression patterns of genes in Nasu-Hakola (NHD) patients and healthy controls (CTR). The heat map indicates up-regulation (red) and down-regulation (green). b Gene ontology analy-

contribute to the defect in removing myelin debris from the tissue [8]. Interestingly, in the current study at this time point no differences in the number of microglia were detected between Trem $2^{+/-}$and Trem $2^{+/+}$mice, despite more myelin debris were accumulating in the CC of Trem $2^{+/-}$. This could suggest that one copy of TREM 2 is sufficient to sustain microglia expansion after CPZ-induced damage, but cannot support a fully functional response by microglia to clear the conspicuous amount of myelin debris derived from massive oligodendrocyte death. Alternatively, it is possible that a defect in microglia proliferation in $T r e m 2^{+/-}$mice is present, but at earlier time points (before 4 weeks) and in a

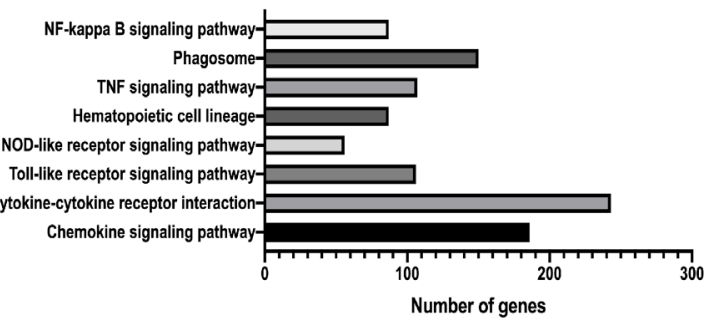

sis of the differentially expressed genes in NHD patients. c Volcano plots showing the distribution of gene expression fold changes in genes of the phagosome pathway. Genes with fold change $>2$ and $P$ value $<0.05$ are indicated in red, and genes with fold change $<-2$ and $P$ value $<0.05$ are indicated in green

very narrow time window. To this end, a very robust microglia activation and increase in density have been shown to start at $2-3$ weeks after $\mathrm{CPZ}$ diet initiation, reaching a plateau at $4-5$ weeks [15]. Future studies are needed to clarify if TREM 2 activation could also directly enhance microglia proliferation in vivo.

TREM2 has been described to be intimately linked to microglia lipid metabolism [8, 42]. Lipids have been proposed as candidates for TREM2 ligands either as free molecules, complexed in myelin or in apolipoprotein particles $[1,2,42,50]$. A recent report demonstrated that TREM 2 is highly expressed in the adipose tissue by lipid-associated 
macrophages (LAM) where it drives gene expression programs involved in phagocytosis and lipid metabolism [19]. LAM cells in adipose tissue indeed expressed a highly similar gene profile as disease-associated microglia in $\mathrm{AD}$ with the exception of few tissue-specific genes [19,21]. Chronic demyelination in vivo (12 weeks on CPZ) caused a robust accumulation of cholesteryl ester (CE) and oxidized CE in the Trem $2^{-/-}$brain, suggesting that TREM 2 might be a crucial transcriptional regulator of cholesterol transport and lipid metabolism in microglia [35]. In support of a strong link between TREM 2 and lipid metabolism are also the original reports describing NHD as a lipid storage disease due to a genetic enzymatic defect leading to lipid and cholesterol accumulation in the brain and bone cysts [37]. Therefore, it is attractive to suggest that a major role for TREM2 in microglia would be intracellular processing and degradation of lipid-rich material (e.g. myelin, cell membranes), especially after extensive demyelination. In our previous work, we provided initial evidence to support this hypothesis by showing that TREM 2 is not a limiting factor in myelin engulfment, but it was clearly essential for microglia capacity to degrade myelin during chronic demyelination [8]. In the current study, we further support this hypothesis and we expand by showing that AL002a treatment significantly increases microglia capacity to phagocyte and degrade myelin in vivo and in vitro. These results confirm TREM2 as a key regulator of phagocytic clearance of myelin debris and lipid metabolism by microglia.

The identification of therapies promoting remyelination is a new frontier to overcome in MS. Approved diseasemodifying treatments for MS are targeting the inflammatory disease component by reducing attack frequency and severity. Currently, no therapies are available to regenerate myelin and to halt MS disease progression. In MS multiple factors are thought to be involved in remyelination failure, starting with a deficiency in OPCs because of impaired recruitment, or incomplete differentiation and maturation in the MS lesions. Several lines of evidence indicate a key role of microglia in these processes. Microglia regenerative expression profile involves genes related to phagocytosis, breakdown of myelin debris, as well as secretion of regenerative factors and tissue remodeling $[36,49]$ that can drive OPC differentiation [29, 32]. Here we show that TREM2 was highly expressed within active MS lesions by lipid-laden microglia/macrophages known to display an alternatively activated $\mathrm{M} 2$ profile and to promote the resolution of inflammation and clearance of myelin debris [5]. AL002a, by targeting and activating mouse TREM2, seems to enhance some of these pathways. It is not fully clear if enhanced remyelination in AL002a-treated Trem $2^{+/-}$mice is due to accelerated removal of myelin debris or if there is an additional TREM2-mediated signal from the microglia to recruit OPCs, thus giving an advantage in remyelination.
Successive studies will further clarify potential microglia polarization or remodeling induced by AL002a. On the other end, it is well known that Trem $2^{+/+}$mouse microglia are very efficient in supporting complete remyelination in the acute CPZ model. This is different from what observed in MS, where a defect in TREM2 pathways has not been demonstrated, but still remyelination fails in most cases. A possible hypothesis could be that, in MS, microglia are chronically challenged by recurring bouts of demyelination and remyelination, suggesting that they might become exhausted and less able to respond over time. In this situation, microglia could be more responsive to an increase in TREM2 signaling via antibody-mediated activation. In future studies we could examine this further by either chronically feeding CPZ or looking in aged mice in which microglia functions could be defective.

Importantly, our study has also shown that AL002a treatment after $\mathrm{CPZ}$ is associated with reduced Nf-L plasma levels (a markers of axonal/neuronal damage), suggesting that increased remyelination also results in preserved axonal integrity, further proven by increased SMI31 staining in the CC of AL002a-treated mice. Altogether our study indicates that strategies aimed at targeting TREM2 on microglia in the CNS are feasible and might be a promising intervention in MS to promote microglia functions in clearing myelin debris, favoring the recruitment of OPCs, and their subsequent differentiation into mature myelin-generating oligodendrocytes, eventually leading to remyelination and axonal protection.

Acknowledgements We are thankful to NHD patients and their families for kindly providing peripheral blood samples for the analyses included in this manuscript. We thank R. C. Paolicelli for her valuable advice with Imaris analysis. This study was supported by grant \# RF1AG058501 (to L.P. and C. Cruchaga) from the National Institute of Aging. Funding for this study was also provided by Alector, Fondazione Italiana Sclerosi Multipla (FISM; 2017/R/20) and co-financed with the ' 5 per mille' public funding, and The Trish MS Research Foundation. C. Cantoni was supported during the course of this study by the National MS Society Career Transition Fellowship (TA-180531003). F.F was supported by a McDonnell Center for Cellular and Molecular Neurobiology (Washington University in St Louis) postdoctoral fellowship and she is the recipient of a 2018-0364-CARIPLO grant. Funding for this project was provided by the Children's Discovery Institute of Washington University and St. Louis Children's Hospital. Experiments/data/analysis/presentation [by the use of a Zeiss LSM880 Airyscan confocal microscope, an Olympus FV1200 confocal microscope, and Imaris Software (Bitplane, Switzerland)] were performed in part through the use of Washington University Center for Cellular Imaging (WUCCI) supported by Washington University School of Medicine, The Children's Discovery Institute of Washington University and St. Louis Children's Hospital (CDI-CORE-2015-505 and CDI-CORE-2019-813) and the Foundation for Barnes-Jewish Hospital (3770 and 4642). Confocal/super-resolution data were generated on a Zeiss LSM 880 Airyscan Confocal Microscope which was purchased with support from the Office of Research Infrastructure Programs (ORIP), a part of the NIH Office of the Director under grant OD021629. 
Author contributions FC and FF designed the study, performed the experiments, analyzed, and interpreted the data. BB, CC carried out the experiments, analyzed and interpreted the data. AL, RM, IA, MM and LD carried out some experiments. DL, BBA, CC, $\mathrm{KM}$ and $\mathrm{OH}$ performed microarray analysis of macrophages from NHD patients and contributed to the interpretation of the data. MB assisted with histological analyses of human MS and control tissues. AR and DMH contributed to the design of the study and interpretation of the data. TS, IT designed the study, performed in vitro experiments, analyzed, and interpreted the data. LP conceived and designed the study, supervised the entire project, the experimental design, data interpretation and manuscript preparation. FF and FC wrote the original draft of the manuscript. FF, BB, FC and IT prepared the figures. All authors edited and reviewed the manuscript.

\section{Compliance with ethical standards}

Conflict of interest IT., T.S., F.C., A.R. and A.I. are Alector employees. L.P. receives research support from Alector. D.M.H. is listed as an inventor on a provisional patent from Washington University on TREM2 antibodies. D.M.H. co-founded and is on the scientific advisory board of $\mathrm{C} 2 \mathrm{~N}$ Diagnostics. Washington University receives research support from C2N Diagnostics on the use of TREM2 antibodies.

Open Access This article is licensed under a Creative Commons Attribution 4.0 International License, which permits use, sharing, adaptation, distribution and reproduction in any medium or format, as long as you give appropriate credit to the original author(s) and the source, provide a link to the Creative Commons licence, and indicate if changes were made. The images or other third party material in this article are included in the article's Creative Commons licence, unless indicated otherwise in a credit line to the material. If material is not included in the article's Creative Commons licence and your intended use is not permitted by statutory regulation or exceeds the permitted use, you will need to obtain permission directly from the copyright holder. To view a copy of this licence, visit http://creativecommons.org/licenses/by/4.0/.

\section{References}

1. Atagi Y, Liu CC, Painter MM, Chen XF, Verbeeck C, Zheng $\mathrm{H}$ et al (2015) Apolipoprotein $\mathrm{E}$ is a ligand for triggering receptor expressed on myeloid cells 2 (TREM2). J Biol Chem 290:26043-26050. https://doi.org/10.1074/jbc.M115.679043

2. Bailey CC, DeVaux LB, Farzan M (2015) The triggering receptor expressed on myeloid cells 2 binds apolipoprotein $\mathrm{E}$. J Biol Chem 290:26033-26042. https://doi.org/10.1074/jbc. M115.677286

3. Bennett ML, Bennett FC, Liddelow SA, Ajami B, Zamanian JL, Fernhoff NB et al (2016) New tools for studying microglia in the mouse and human CNS. Proc Natl Acad Sci USA 113:E1738E1746. https://doi.org/10.1073/pnas.1525528113

4. Bock V, Botturi A, Gaviani P, Lamperti E, Maccagnano C, Piccio L et al (2013) Polycystic lipomembranous osteodysplasia with sclerosing leukoencephalopathy (PLOSL): a new report of an Italian woman and review of the literature. J Neurol Sci 326:115-119. https://doi.org/10.1016/j.jns.2013.01.021

5. Boven LA, Van Meurs M, Van Zwam M, Wierenga-Wolf A, Hintzen RQ, Boot RG et al (2006) Myelin-laden macrophages are anti-inflammatory, consistent with foam cells in multiple sclerosis. Brain 129:517-526

6. Butovsky O, Jedrychowski MP, Moore CS, Cialic R, Lanser AJ, Gabriely G et al (2014) Identification of a unique
TGF-beta-dependent molecular and functional signature in microglia. Nat Neurosci 17:131-143. https://doi.org/10.1038/nn.3599

7. Cady J, Koval ED, Benitez BA, Zaidman C, Jockel-Balsarotti J, Allred $\mathrm{P}$ et al (2014) TREM2 variant $\mathrm{p}$. R47H as a risk factor for sporadic amyotrophic lateral sclerosis. JAMA Neurol 71:449-453. https://doi.org/10.1001/jamaneurol.2013.6237

8. Cantoni C, Bollman B, Licastro D, Xie M, Mikesell R, Schmidt $\mathrm{R}$ et al (2015) TREM2 regulates microglial cell activation in response to demyelination in vivo. Acta Neuropathol 129:429447. https://doi.org/10.1007/s00401-015-1388-1

9. Cella M, Buonsanti C, Strader C, Kondo T, Salmaggi A, Colonna M (2003) Impaired differentiation of osteoclasts in TREM-2-deficient individuals. J Exp Med 198:645-651

10. Daws MR, Sullam PM, Niemi EC, Chen TT, Tchao NK, Seaman WE (2003) Pattern recognition by TREM-2: binding of anionic ligands. J Immunol 171:594-599

11. Dulamea AO (2017) Role of oligodendrocyte dysfunction in demyelination, remyelination and neurodegeneration in multiple sclerosis. Adv Exp Med Biol 958:91-127. https://doi. org/10.1007/978-3-319-47861-6_7

12. Eskelinen EL, Tanaka Y, Saftig P (2003) At the acidic edge: emerging functions for lysosomal membrane proteins. Trends Cell Biol 13:137-145. https://doi.org/10.1016/s0962-8924(03)00005-9

13. Forabosco P, Ramasamy A, Trabzuni D, Walker R, Smith C, Bras $\mathrm{J}$ et al (2013) Insights into TREM2 biology by network analysis of human brain gene expression data. Neurobiol Aging 34:26992714. https://doi.org/10.1016/j.neurobiolaging.2013.05.001

14. Franklin RJM, Ffrench-Constant C (2017) Regenerating CNS myelin-from mechanisms to experimental medicines. Nat Rev Neurosci 18:753-769. https://doi.org/10.1038/nrn.2017.136

15. Gudi V, Gingele S, Skripuletz T, Stangel M (2014) Glial response during cuprizone-induced de- and remyelination in the CNS: lessons learned. Front Cell Neurosci 8:73. https://doi.org/10.3389/ fncel.2014.00073

16. Guerreiro R, Wojtas A, Bras J, Carrasquillo M, Rogaeva E, Majounie E et al (2012) TREM2 variants in Alzheimer's disease. N Engl J Med 368:117-127. https://doi.org/10.1056/NEJMoa1211 851

17. Hiremath MM, Saito Y, Knapp GW, Ting JP, Suzuki K, Matsushima GK (1998) Microglial/macrophage accumulation during cuprizone-induced demyelination in C57BL/6 mice. J Neuroimmunol 92:38-49

18. Irvine KA, Blakemore WF (2008) Remyelination protects axons from demyelination-associated axon degeneration. Brain 131:1464-1477. https://doi.org/10.1093/brain/awn080

19. Jaitin DA, Adlung L, Thaiss CA, Weiner A, Li B, Descamps H et al (2019) Lipid-associated macrophages control metabolic homeostasis in a Trem2-dependent manner. Cell 178(686698):e614. https://doi.org/10.1016/j.cell.2019.05.054

20. Jonsson T, Stefansson H, Steinberg S, Jonsdottir I, Jonsson PV, Snaedal J et al (2012) Variant of TREM2 associated with the risk of Alzheimer's disease. N Engl J Med 368:107-116. https://doi. org/10.1056/NEJMoa1211103

21. Keren-Shaul H, Spinrad A, Weiner A, Matcovitch-Natan O, Dvir-Szternfeld R, Ulland TK et al (2017) A unique microglia type associated with restricting development of Alzheimer's disease. Cell 169(1276-1290):e1217. https://doi.org/10.1016/j. cell.2017.05.018

22. Kiernan JA (1984) Chromoxane cyanine R. II. Staining of animal tissues by the dye and its iron complexes. J Microsc 134:25-39

23. Kiialainen A, Hovanes K, Paloneva J, Kopra O, Peltonen L (2005) Dap12 and Trem2, molecules involved in innate immunity and neurodegeneration, are co-expressed in the CNS. Neurobiol Dis $18: 314-322$

24. Kleinberger G, Yamanishi Y, Suarez-Calvet M, Czirr E, Lohmann E, Cuyvers E et al (2014) TREM2 mutations implicated in 
neurodegeneration impair cell surface transport and phagocytosis. Sci Transl Med 6:243ra286. https://doi.org/10.1126/scitranslm ed.3009093

25. Klunemann HH, Ridha BH, Magy L, Wherrett JR, Hemelsoet DM, Keen RW et al (2005) The genetic causes of basal ganglia calcification, dementia, and bone cysts: DAP12 and TREM2. Neurology 64:1502-1507

26. Kotter MR, Li WW, Zhao C, Franklin RJ (2006) Myelin impairs CNS remyelination by inhibiting oligodendrocyte precursor cell differentiation. J Neurosci Off J Soc Neurosci 26:328-332. https ://doi.org/10.1523/JNEUROSCI.2615-05.2006

27. Lampron A, Larochelle A, Laflamme N, Prefontaine P, Plante MM, Sanchez MG et al (2015) Inefficient clearance of myelin debris by microglia impairs remyelinating processes. J Exp Med 212:481-495. https://doi.org/10.1084/jem.20141656

28. Lassmann H, Bruck W, Lucchinetti CF (2007) The immunopathology of multiple sclerosis: an overview. Brain Pathol 17:210-218. https://doi.org/10.1111/j.1750-3639.2007.00064.x

29. Lloyd AF, Miron VE (2019) The pro-remyelination properties of microglia in the central nervous system. Nat Rev Neurol 15:447458. https://doi.org/10.1038/s41582-019-0184-2

30. Matsuo A, Lee GC, Terai K, Takami K, Hickey WF, McGeer EG et al (1997) Unmasking of an unusual myelin basic protein epitope during the process of myelin degeneration in humans: a potential mechanism for the generation of autoantigens. Am J Pathol 150:1253-1266

31. Matsushima GK, Morell P (2001) The neurotoxicant, cuprizone, as a model to study demyelination and remyelination in the central nervous system. Brain Pathol 11:107-116

32. Miron VE, Boyd A, Zhao JW, Yuen TJ, Ruckh JM, Shadrach JL et al (2013) M2 microglia and macrophages drive oligodendrocyte differentiation during CNS remyelination. Nat Neurosci 16:1211-1218. https://doi.org/10.1038/nn.3469

33. Neumann H, Kotter MR, Franklin RJ (2009) Debris clearance by microglia: an essential link between degeneration and regeneration. Brain 132:288-295. https://doi.org/10.1093/brain/awn109

34. Norton WT (1974) Isolation of myelin from nerve tissue. Methods Enzymol 31:435-444. https://doi.org/10.1016/00766879(74)31049-x

35. Nugent AA, Lin K, van Lengerich B, Lianoglou S, Przybyla L, Davis SS et al (2019) TREM2 regulates microglial cholesterol metabolism upon chronic phagocytic challenge. Neuron. https:// doi.org/10.1016/j.neuron.2019.12.007

36. Olah M, Amor S, Brouwer N, Vinet J, Eggen B, Biber K et al (2012) Identification of a microglia phenotype supportive of remyelination. Glia 60:306-321. https://doi.org/10.1002/glia.21266

37. Paloneva J, Autti T, Raininko R, Partanen J, Salonen O, Puranen $M$ et al (2001) CNS manifestations of Nasu-Hakola disease: a frontal dementia with bone cysts. Neurology 56:1552-1558

38. Paloneva J, Kestila M, Wu J, Salminen A, Bohling T, Ruotsalainen $\mathrm{V}$ et al (2000) Loss-of-function mutations in TYROBP (DAP12) result in a presenile dementia with bone cysts. Nat Genet 25:357361. https://doi.org/10.1038/77153

39. Paloneva J, Manninen T, Christman G, Hovanes K, Mandelin J, Adolfsson R et al (2002) Mutations in two genes encoding different subunits of a receptor signaling complex result in an identical disease phenotype. Am J Hum Genet 71:656-662

40. Pawitan Y, Michiels S, Koscielny S, Gusnanto A, Ploner A (2005) False discovery rate, sensitivity and sample size for microarray studies. Bioinformatics 21:3017-3024. https://doi.org/10.1093/ bioinformatics/bti448

41. Piccio L, Buonsanti C, Cella M, Tassi I, Schmidt RE, Fenoglio $\mathrm{C}$ et al (2008) Identification of soluble TREM-2 in the cerebrospinal fluid and its association with multiple sclerosis and CNS inflammation. Brain 131:3081-3091. https://doi.org/10.1093/brain /awn217

42. Poliani PL, Wang Y, Fontana E, Robinette ML, Yamanishi Y, Gilfillan $\mathrm{S}$ et al (2015) TREM2 sustains microglial expansion during aging and response to demyelination. J Clin Investig 125:21612170. https://doi.org/10.1172/JCI77983

43. Rayaprolu S, Mullen B, Baker M, Lynch T, Finger E, Seeley WW et al (2013) TREM2 in neurodegeneration: evidence for association of the p.R47H variant with frontotemporal dementia and Parkinson's disease. Mol Neurodegener 8:19. https://doi. org/10.1186/1750-1326-8-19

44. Schafer DP, Lehrman EK, Heller CT, Stevens B (2014) An engulfment assay: a protocol to assess interactions between CNS phagocytes and neurons. J Vis Exp. https://doi.org/10.3791/51482

45. Schmid CD, Sautkulis LN, Danielson PE, Cooper J, Hasel KW, Hilbush BS et al (2002) Heterogeneous expression of the triggering receptor expressed on myeloid cells- 2 on adult murine microglia. J Neurochem 83:1309-1320

46. Takahashi K, Rochford CD, Neumann H (2005) Clearance of apoptotic neurons without inflammation by microglial triggering receptor expressed on myeloid cells-2. J Exp Med 201:647-657

47. Ulland TK, Colonna M (2018) TREM2-a key player in microglial biology and Alzheimer disease. Nat Rev Neurol 14:667-675. https://doi.org/10.1038/s41582-018-0072-1

48. Voet S, Prinz M, van Loo G (2019) Microglia in central nervous system inflammation and multiple sclerosis pathology. Trends Mol Med 25:112-123. https://doi.org/10.1016/j.molmed.2018.11.005

49. Voss EV, Skuljec J, Gudi V, Skripuletz T, Pul R, Trebst C et al (2012) Characterisation of microglia during de- and remyelination: can they create a repair promoting environment? Neurobiol Dis 45:519-528. https://doi.org/10.1016/j.nbd.2011.09.008

50. Wang Y, Cella M, Mallinson K, Ulrich JD, Young KL, Robinette ML et al (2015) TREM2 lipid sensing sustains the microglial response in an Alzheimer's disease model. Cell 160:1061-1071. https://doi.org/10.1016/j.cell.2015.01.049

51. Wang Y, Ulland TK, Ulrich JD, Song W, Tzaferis JA, Hole JT et al (2016) TREM2-mediated early microglial response limits diffusion and toxicity of amyloid plaques. J Exp Med 213:667-675. https://doi.org/10.1084/jem.20151948

52. Wei SH, Parker I, Miller MJ, Cahalan MD (2003) A stochastic view of lymphocyte motility and trafficking within the lymph node. Immunol Rev 195:136-159

53. Yuan P, Condello C, Keene CD, Wang Y, Bird TD, Paul SM et al (2016) TREM2 haplodeficiency in mice and humans impairs the microglia barrier function leading to decreased amyloid compaction and severe axonal dystrophy. Neuron 92:252-264. https://doi. org/10.1016/j.neuron.2016.09.016

Publisher's Note Springer Nature remains neutral with regard to jurisdictional claims in published maps and institutional affiliations. 